\title{
The evolution of the hard X-ray luminosity function of AGN
}

\author{
J. Aird, ${ }^{1 \star} \dagger$ K. Nandra, ${ }^{1}$ E. S. Laird, ${ }^{1}$ A. Georgakakis, ${ }^{2}$ M. L. N. Ashby, ${ }^{3}$ P. Barmby, ${ }^{4}$ \\ A. L. Coil, ${ }^{5}$ J.-S. Huang, ${ }^{3}$ A. M. Koekemoer, ${ }^{6}$ C. C. Steidel $^{7}$ and C. N. A. Willmer ${ }^{8}$ \\ ${ }^{1}$ Astrophysics Group, Imperial College London, Blackett Laboratory, Prince Consort Road, London SW7 2AZ \\ ${ }^{2}$ National Observatory of Athens, Institute of Astronomy, V. Paulou \& I. Metaxa, Athens 15236, Greece \\ ${ }^{3}$ Harvard-Smithsonian Center for Astrophysics, 60 Garden Street, Cambridge, MA 02138, USA \\ ${ }^{4}$ Department of Physics \& Astronomy, University of Western Ontario, London, ON N6A 3K7, Canada \\ ${ }^{5}$ Center for Astrophysics and Space Sciences (CASS), Department of Physics, University of California, San Diego, CA 92093, USA \\ ${ }^{6}$ Space Telescope Science Institute, 3700 San Martin Drive, Baltimore MD 21218, USA \\ ${ }^{7}$ California Institute of Technology, MS 105-24, Pasadena, CA 91125, USA \\ ${ }^{8}$ Steward Observatory, University of Arizona, Tucson, AZ 85721, USA
}

Accepted 2009 October 5. Received 2009 September 3; in original form 2009 April 29

\begin{abstract}
We present new observational determinations of the evolution of the 2-10 keV X-ray luminosity function (XLF) of active galactic nuclei (AGN). We utilize data from a number of surveys including both the 2 Ms Chandra Deep Fields and the AEGIS-X 200 ks survey, enabling accurate measurements of the evolution of the faint end of the XLF. We combine direct, hard $\mathrm{X}$-ray selection and spectroscopic follow-up or photometric redshift estimates at $z<1.2$ with a rest-frame UV colour pre-selection approach at higher redshifts to avoid biases associated with catastrophic failure of the photometric redshifts. Only robust optical counterparts to X-ray sources are considered using a likelihood ratio matching technique. A Bayesian methodology is developed that considers redshift probability distributions, incorporates selection functions for our high-redshift samples and allows robust comparison of different evolutionary models. We statistically account for X-ray sources without optical counterparts to correct for incompleteness in our samples. We also account for Poissonian effects on the X-ray flux estimates and sensitivities and thus correct for the Eddington bias. We find that XLF retains the same shape at all redshifts, but undergoes strong luminosity evolution out to $z \sim 1$, and an overall negative density evolution with increasing redshift, which thus dominates the evolution at earlier times. We do not find evidence that a luminosity-dependent density evolution, and the associated flattening of the faint-end slope, is required to describe the evolution of the XLF. We find significantly higher space densities of low-luminosity, high-redshift AGN than in prior studies, and a smaller shift in the peak of the number density to lower redshifts with decreasing luminosity. The total luminosity density of AGN peaks at $z=1.2 \pm 0.1$, but there is a mild decline to higher redshifts. We find that $>50$ per cent of black hole growth takes place at $z>1$, with around half in $L_{\mathrm{X}}<10^{44} \mathrm{erg} \mathrm{s}^{-1}$ AGN.
\end{abstract}

Key words: galaxies: active - galaxies: evolution - galaxies: luminosity function, mass function - X-rays: galaxies.

\section{INTRODUCTION}

Active galactic nuclei (AGN) are an important constituent of the Universe, playing a vital role in the formation and evolution of galaxies and having a wider influence on the structure of the Universe. Determining the distribution and evolution of AGN accretion

^E-mail: jaird@ucsd.edu

$\nmid$ Present address: Center for Astrophysics and Space Sciences (CASS), Department of Physics, University of California, San Diego, CA 92093, USA. activity throughout the history of the Universe, traced by the luminosity function, is essential to constrain models of super-massive black hole formation and growth, the triggering and fuelling of AGN and their co-evolution with galaxies. This requires large samples of objects spanning a wide range of redshifts and luminosities to accurately determine the shape of the luminosity function and how this evolves over cosmic time.

X-ray surveys provide a highly efficient method of selecting AGN, including unobscured and moderately obscured sources and low-luminosity sources that may not be identified as AGN at optical wavelengths. X-ray emission is relatively unaffected by absorption, 
particularly at hard X-ray energies ( $\gtrsim 2 \mathrm{keV}$ ) for moderate column densities $\left(N_{\mathrm{H}} \lesssim 10^{23} \mathrm{~cm}^{-2}\right)$, and thus provides a direct probe of the accretion activity. Large efforts have been made to obtain follow-up observations of X-ray sources in various X-ray surveys to determine their redshifts, and thus allow the X-ray luminosity function (XLF) to be determined (e.g. Barger et al. 2003; Szokoly et al. 2004; Trouille et al. 2008). Such studies of the XLF have revealed that AGN are a strongly evolving population, with the overall distribution shifting to lower luminosities between $z \sim 1$ and the present day. This evolution can be described by a pure luminosity evolution (PLE) parametrization (Boyle et al. 1993; Barger et al. 2005) in which the XLF retains the same shape (a double power law with a break at a characteristic luminosity, $L_{*}$ ), but shifts to lower luminosities as redshift decreases. However, recent data indicate that a more complex, luminosity-dependent density evolution (LDDE) parametrization is required to describe the evolution of the XLF at both low $(z \lesssim 1)$ and high $(z \approx 1-4)$ redshifts (e.g. Ueda et al. 2003; La Franca et al. 2005; Silverman et al. 2008; Ebrero et al. 2009; Yencho et al. 2009). In this scheme the shape of the XLF changes with redshift, with the faint-end slope flattening as redshift increases. This evolution is also characterized by a shift in the peak of the space density of AGN towards lower redshifts for lower luminosities.

However, there are remaining uncertainties as to the exact form of the evolution, especially at high redshifts. In part, this is due to the difficulties of accurately measuring the faint end of the XLF, where incomplete and uncertain redshift information is a serious issue. The Chandra Deep Fields-North (CDF-N; Alexander et al. 2003) and Chandra Deep Fields-South (CDF-S; Giacconi et al. 2002) have been key in studies of the XLF, providing the deepest available X-ray data and substantial programmes of spectroscopic follow-up of the X-ray sources to determine redshifts. However, even in these fields a high fraction ( $\sim 50$ per cent) of the X-ray sources remains spectroscopically unidentified, mainly because the optical counterparts of the faintest $\mathrm{X}$-ray sources are also faint $(R \gtrsim$ 24 ), and thus beyond the limits of current instrumentation. This can severely bias determinations of the XLF, especially at high redshifts (e.g. Barger et al. 2005).

One general approach to address this issue is to determine photometric redshifts for the X-ray sources that are too faint for optical spectroscopic follow-up. This significantly improves the completeness of the samples, although many X-ray sources which lack detectable optical counterparts remain unidentified. However, there are considerable uncertainties in such redshift estimates, particularly for the faintest optical counterparts, and a risk of catastrophic failures in the redshift determinations, which is particularly acute for sources at $z \sim 1-3$. It is also necessary to correct for the remaining incompleteness in such samples, which can prove difficult. Additionally, there is a significant risk of associating the incorrect optical counterpart with an X-ray source, due to the high surface density of optical sources at these faint magnitudes, and thus an $\mathrm{X}$-ray source may be assigned completely incorrect redshift information. For example, Barger et al. (2003) were able to assign optical counterparts to $\sim 85$ per cent of the X-ray sources in the CDF-N, but noted that $\sim 25$ per cent of the optical identifications at $R=24-26$ are expected to be spurious. Such false identifications of faint X-ray sources could significantly bias determinations of the XLF. To reduce these effects many authors (e.g. Ueda et al. 2003; Barger et al. 2005; Silverman et al. 2008) set relatively high X-ray flux limits, thus increasing the spectroscopically identified fraction and reducing the numbers of optically faint or unidentified sources, but this also reduces the sensitivity to the key low-luminosity AGN. The small number of fields with sufficiently high spectroscopic completeness also means that most results are susceptible to cosmic variance.

An alternative approach to the incompleteness issue was adopted by Aird et al. (2008) (see also Nandra et al. 2005) to determine the XLF at high redshift, where the effects can be most severe. The Lyman-break technique (e.g. Steidel \& Hamilton 1993; Steidel et al. 2003) was used to pre-select a sample of objects at $z \sim 3$ based on their broad-band colours in three filters, and deep X-ray observations were used to identify AGN. This technique provides an incomplete sample (missing heavily reddened sources in particular), but the optical selection functions are well defined and were derived using simulations of the optical data. This allowed corrections for the incompleteness to be applied, and thus the faint end of the XLF at $z \sim 3$ could be accurately measured. The number density of low-luminosity AGN was at least as high as previous estimates, and there was no evidence for the flattening of the faint-end slope at $z>1$ that is characteristic of LDDE parametrizations. However, this work did not account for the uncertainties in the redshift estimates for sources without spectroscopic confirmation and did not present an evolutionary model.

In addition to uncertainties in the optical follow-up and redshift estimates, a number of issues arising from the X-ray observations may also affect the determination of the evolution of the XLF. The faintest sources in Chandra surveys are detected with very small numbers of counts, and thus Poissonian effects can be significant. Derived X-ray fluxes can thus be highly uncertain, and may be underestimated by up to 50 per cent due to the Eddington bias (Laird et al. 2009), leading to uncertainties in the luminosity. Georgakakis et al. (2008) presented a new method of determining the sensitivity of Chandra observations, which fully accounts for the Poissonian nature of the detection limits and accounted for the flux uncertainties and Eddington bias in the determination of the X-ray number counts. However, these techniques have not been incorporated in previous studies of the XLF.

In this paper we present a new study of the evolution of the XLF, in which we incorporate new deep X-ray observations and develop improved methodologies to address some of the issues regarding optical counterparts, completeness, photometric redshifts and X-ray fluxes described above. Section 2 describes our data, including our samples of hard X-ray-selected AGN and the data used to perform high-redshift colour pre-selection of AGN. We adopt a likelihood ratio method to ensure that we assign secure optical counterparts to the faintest X-ray sources. In Section 3 we develop a Bayesian methodology to determine the evolution of the XLF, which accounts for uncertainties in redshift estimates and $\mathrm{X}$-ray fluxes, incorporates the improved sensitivity calculations of Georgakakis et al. (2008), corrects for the remaining incompleteness of the optical identifications and allows a robust comparison of different evolutionary models. Section 3.3 describes how highredshift rest-frame UV colour-selected samples are incorporated into this methodology (building on the work of Aird et al. 2008), to provide a more robust determination of the evolution at $z \gtrsim 1.2$. Our results are presented in Section 4 and discussed further in Section 5.

A flat cosmology with $\Omega_{\Lambda}=0.7$ and $h=0.7$ is adopted throughout.

\section{DATA AND SAMPLES}

To determine the evolution of the XLF requires large samples of AGN spanning a wide range of luminosities and redshifts. For our initial investigation of the evolution of the XLF, our approach is 
Table 1. Details of the hard X-ray-selected samples/fields.

\begin{tabular}{|c|c|c|c|c|c|c|c|}
\hline Field & $\begin{array}{c}\text { RA } \\
(\mathrm{J} 2000)\end{array}$ & $\begin{array}{l}\text { Dec. } \\
(\mathrm{J} 2000)\end{array}$ & $\begin{array}{l}\text { X-ray exposure } \\
(\mathrm{ks})\end{array}$ & $\begin{array}{l}\text { Survey area }{ }^{a} \\
\left(\operatorname{arcmin}^{2}\right)\end{array}$ & $N_{\mathrm{X}}^{b}$ & $\begin{array}{c}F_{\text {opt }}{ }^{c} \\
\text { (per cent) }\end{array}$ & $\begin{array}{c}F_{\text {spec }}^{d} \\
\text { (per cent) }\end{array}$ \\
\hline CDF-S & $03: 32: 28$ & $-27: 48: 30$ & 1933.4 & 436.2 & 248 & 68 & 44 \\
\hline CDF-N & $12: 36: 55$ & $+62: 14: 18$ & 1862.9 & 436.0 & 303 & 67 & 55 \\
\hline AEGIS-X & $14: 17: 43$ & $+52: 28: 25$ & 188.7 & 1155.6 & 397 & 86 & 40 \\
\hline ALSS & 13:14:00 & $+31: 30: 00$ & - & 20880.0 & 34 & 97 & 97 \\
\hline AMSS & - & - & - & 294386.0 & 109 & 98 & 98 \\
\hline
\end{tabular}

${ }^{a}$ Area covered by both X-ray data and required optical imaging; ${ }^{b}$ total number of hard X-ray-selected sources in the area covered; ${ }^{c}$ fraction of hard X-ray sources with secure optical counterparts; ${ }^{d}$ fraction of hard X-ray sources with secure optical counterparts and spectroscopic redshifts.

similar to most previous studies of the XLF (e.g. Ueda et al. 2003; Barger et al. 2005; Silverman et al. 2008; Ebrero et al. 2009) in that we attempt to assign redshifts to all sources in a purely $\mathrm{X}$-ray-selected catalogue, utilizing photometric redshifts to improve the completeness. Thus AGN are detected over a wide redshift range, which is essential for probing the evolution. Our methodology (see Section 3) requires probability distributions that describe the uncertainties in the photometric redshift estimates. We therefore adopt the best available photometric redshifts with suitable probability distributions or generate our own from the available data. To obtain the best constraints on the XLF, a high fraction of spectroscopic identifications is also required. Additionally, we require hard X-ray coverage to reduce biases against moderately absorbed sources. Three deep Chandra surveys have the necessary spectroscopic follow-up, combined with the deep multiwavelength imaging required for photometric redshifts: the two $\mathrm{CDF}$ and the AEGIS-X survey. We supplement this sample with two larger area ASCA surveys with near complete spectroscopic follow-up, which are needed to constrain the bright end of the XLF. Table 1 summarizes the numbers of sources and fraction with optical counterparts and spectroscopic redshifts. Further details and a description of the photometric redshift estimates are given in Section 2.1.

For the high-redshift colour pre-selection approach, the requirements are different. Deep X-ray data are again required, but the soft $(0.5-2 \mathrm{keV})$ band, where the sensitivity of Chandra is highest, may be used for selection as this probes relatively hard rest-frame energies at $z \gtrsim 2$, and thus should not be severely biased by absorption effects. Deep optical imaging data in $U_{n} \mathrm{G} \mathcal{R}$ or a comparable filter set are required for the colour pre-selection, with deep data at $U$-band wavelengths being the major requirement. A description of the data for the high-redshift work is given in Section 2.2.

\subsection{Hard X-ray-selected samples of AGN}

\subsubsection{Chandra Deep Field-South}

The original $\sim 1$ Ms observation of the CDF-S (Giacconi et al. 2002) has recently been supplemented with additional Director's Discretionary Time to provide a total of $\sim 2 \mathrm{Ms}$ exposure (Luo et al. 2008), which we use in this paper. Data reduction and source detection were carried out using the procedures described in Laird et al. (2009). For our investigation of the XLF, we use the hard $(2-7 \mathrm{keV})$ selected source catalogue of objects detected with the false Poisson probability of $<4 \times 10^{-6}$, which provides a sample of $248 \mathrm{X}$-ray sources. The X-ray sensitivity (area curve) is determined using the procedure described in Georgakakis et al. (2008).

Deep optical data have been obtained in several bands in the CDF$\mathrm{S}$ using both ground-based observatories and the Hubble Space
Telescope (HST). We have searched for optical counterparts using the COMBO-17 survey (Wolf et al. 2004) source catalogue, in which sources are detected in deep $R$-band images $(10 \sigma$ limiting $\mathrm{AB}$ magnitude of $\sim 25.6$ ) obtained with the WFI instrument on the European Southern Observatory (ESO) $2.2 \mathrm{~m}$ telescope at La Silla. Counterparts were found using the implementation of the likelihood ratio method (Sutherland \& Saunders 1992; Ciliegi et al. 2003) described in Laird et al. (2009). This method allows us to assign secure optical counterparts to the X-ray source, accounting for the X-ray and optical positional uncertainties, the a priori probability of a counterpart with a given magnitude and the probability of a spurious optical counterpart. We restrict our sample to objects with likelihood ratios of $>0.5$, which provides optical counterparts for $169(\approx 68$ per cent) of the hard-band-selected X-ray sources.

A number of spectroscopic programmes have been carried out in the CDF-S. Following Luo et al. (2008), we have combined spectroscopic surveys from Szokoly et al. (2004), Le Fèvre et al. (2004), Mignoli et al. (2005), Ravikumar et al. (2007), Popesso et al. (2009) and Vanzella et al. (2008). This provides spectroscopic redshifts for 110 sources (65 per cent of the optical counterparts and 44 per cent of the entire hard X-ray-selected sample).

A variety of photometric redshift estimates have been published in the CDF-S area, focused on both the X-ray source population (e.g. Zheng et al. 2004) and larger populations of optically selected sources (e.g. Mobasher et al. 2004; Wolf et al. 2004). However, we have chosen to use our own photometric redshift estimates using the Bayesian photometric redshift code BPZ (Benítez 2000), which allows us to use the full redshift probability distribution output by the BPZ code and thus account for the significant uncertainties in photo- $z$ estimates in our determination of the XLF (see Section 3). Such probability distributions are not available for the published catalogues.

Our photo- $z$ estimates were determined using the full 17-band photometry for the X-ray sources (with secure optical counterparts) from the COMBO-17 catalogues (Wolf et al. 2004). The BPZ code uses a $\chi^{2}$ minimization template fitting approach. A set of six template spectra are used, consisting of the four standard templates from Coleman, Wu \& Weedman (1980) (E/S0, Sbc, Scd and Irr) and two starbursting galaxy templates from Kinney et al. (1996). Additional points in colour space are calculated by interpolating between the templates. The BPZ code also adopts a Bayesian approach, applying priors based on the expected fractions and redshift distributions for different templates and marginalizing over the templates to produce the final redshift probability distribution, $p(z)$. These distributions describe the uncertainties in the redshift estimate due to both photometric errors and colour, redshift and template degeneracies, which often result in multimodal probability distributions. We note that BPZ does not include AGN or quasi-stellar object (QSO) templates. 


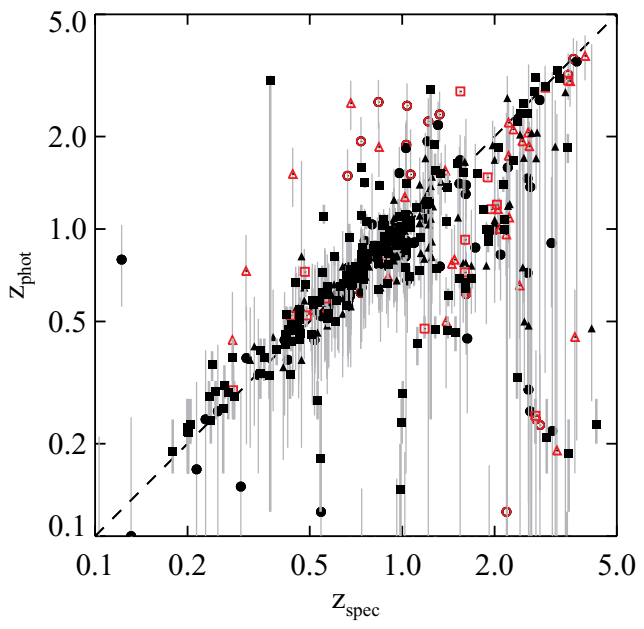

Figure 1. Comparison of spectroscopic and template fitting photometric redshift estimates in the CDF-S (circles), CDF-N (triangles) and AEGIS (squares). Error bars are 95 per cent central confidence intervals derived from the redshift probability distributions. At $z \lesssim 1.0$, the photometric and spectroscopic redshifts are generally in good agreement. At higher redshifts the photo-zs are less reliable, reflected by the larger error bars. A high fraction of the outliers is associated with broad-line QSOs (open symbols), which are poorly fitted by the templates. However, there is a systematic bias to assign $z \gtrsim 1.2$ sources to lower redshifts.

Broad-line QSOs are likely to be optically bright, and may be more easily identified spectroscopically due to their clear spectroscopic features. The remaining AGN population is expected to be fainter, with a significant fraction of the optical light coming from the host galaxy, thus improving the match to the galaxy templates. Uncertainties due to the mismatch between the observed AGN spectral energy distributions (SEDs) and the galaxy templates may be partially reflected in the redshift probability distributions, although the lack of a representative template may result in catastrophic failures, and an unrepresentative $p(z)$. Additionally, the priors are based on observations of the galaxy population in the Hubble Deep Field-North (HDF-N), and thus may not truly represent the AGN population.

In Fig. 1 we compare the available spectroscopic redshifts with the best photo- $z$ estimates, given by the mode of the $p(z)$ distribution. Error bars on the photo- $z$ represent 95 per cent central confidence intervals determined from the $p(z)$ distribution, although will not fully represent more complicated multimodal distributions. The estimates generally agree well at $z_{\text {spec }}<1.5$ and have a $\delta z=\mid\left(z_{\text {phot }}-\right.$ $\left.z_{\text {spec }}\right) \mid /\left(1+z_{\text {spec }}\right) \approx 0.18$. There are however a number of outliers. A high fraction of these are associated with broad-line QSOs, in which the optical light is dominated by the AGN, and thus cannot be accurately described with the BPZ galaxy templates. Excluding these sources reduces the error to $\delta z \approx 0.13$. The remaining outliers are generally at high redshifts $\left(z_{\text {spec }} \gtrsim 1.2\right)$, and are assigned large errors, indicating broad $p(z)$ distributions. However, we note a systematic bias that assigns $z_{\text {spec }} \gtrsim 1.2$ sources to lower redshifts. The $p(z)$ distributions are equally affected by such bias; accounting for the redshift uncertainty, however broad, will not correct for this effect. Indeed, the systematic bias indicates a catastrophic failure of the BPZ code to correctly fit the observed SEDs with realistic templates at the higher redshifts. This failure may severely bias any determination of the XLF. We explore this in more detail in Section 3.3.

\subsubsection{Chandra Deep Field-North}

The CDF-N provides a second $2 \mathrm{Ms} \mathrm{X}$-ray field. A point source catalogue based on the full $2 \mathrm{Ms}$ exposure obtained has been presented by Alexander et al. (2003); however we use our own reduction and source catalogue, again using the procedure described in Laird et al. (2009). Our catalogue probes to lower significances than the Alexander et al. (2003) work and allows us to adopt our improved sensitivity calculations (Georgakakis et al. 2008). In this paper we use objects with significant detections (false Poisson probability of $<4 \times 10^{-6}$ ) in the hard band, providing a sample of $303 \mathrm{X}$-ray sources.

A vast quantity of multiwavelength data are available in this thoroughly observed field (e.g. Dickinson et al. 2003). To find optical counterparts to our X-ray sources, we use the Hawaii HDF-N data (Capak et al. 2004), which fully covers the X-ray data with deep observations in $U, B, V, R, I, z^{\prime}$ and $H K^{\prime}$ bandpasses. Optical counterparts were found using the likelihood ratio method, as in the CDF-S. This yields secure optical counterparts for 204 ( $\approx 67$ per cent) of our hard-band-selected X-ray sources.

We searched for spectroscopic redshifts for our secure optical counterparts in a variety of spectroscopic surveys that have been carried out in the region (Steidel et al. 2003; Reddy et al. 2006; Weiner et al. 2006; Barger, Cowie \& Wang 2008; Trouille et al. 2008). This provided spectroscopic redshifts of 166 sources (81 per cent of the X-ray sources with secure optical counterparts and 55 per cent of the total hard X-ray-selected sample). The spectroscopic completeness is much higher ( $\sim 80$ per cent $)$ for the brightest X-ray sources $\left(f_{\mathrm{X}}>10^{-14} \mathrm{erg} \mathrm{s}^{-1} \mathrm{~cm}^{-2}\right.$; see Trouille et al. 2008).

As for the CDF-S, we have recalculated photometric redshift estimates with BPZ, using the Capak et al. (2004) $U, B, V, R, I, z^{\prime}$ and $H K^{\prime}$ photometry. A comparison of photometric and spectroscopic redshifts is shown in Fig. 1. Again, a large number of the outliers are associated with broad-line AGN, in addition to high-redshift sources which are assigned large errors. Excluding QSOs, the accuracy of our photo-zs is $\delta z \approx 0.11$. A systematic bias at $z_{\text {spec }} \gtrsim 1.2$, assigning sources to lower redshifts, is present, as found for the CDF-S.

\subsubsection{AEGIS}

AEGIS (Davis et al. 2007) is an international multiwavelength survey of the Extended Groth Strip, covering an $\sim 0.25$ by $2^{\circ}$ strip of the sky. Deep ( $\sim 200 \mathrm{ks})$ X-ray data have been obtained along the entire length of the strip. Laird et al. (2009) described the reduction of these data and presented a catalogue of 1325 unique point sources, merged over all bands. In this paper we restrict our analysis to hard-band-detected sources (false Poisson probability of $<4$ $\left.\times 10^{-6}\right)$ and those which also fall within the area with very deep $u^{*} g^{\prime} r^{\prime} i^{\prime} z$ optical data from the Canada-France-Hawaii Telescope Legacy Survey (CFHTLS). ${ }^{1}$ This reduces the sample to 397 X-ray sources, of which 343 (86 per cent) have secure $i^{\prime}$-band optical counterparts (likelihood ratio of $>0.5$ ).

Significant spectroscopic follow-up is available in the AEGIS field, including redshifts from the DEEP2 survey (Davis et al. 2007) and additional targeted follow-up of X-ray sources with MMT (Coil et al. 2009). This provides spectroscopic redshifts for 157 sources (40 per cent).

In this field, we have chosen to use photometric redshifts from two different sources. Huang et al. (in preparation) use an Artificial

\footnotetext{
${ }^{1}$ http://www.cfht.hawaii.edu/Science/CFHTLS
} 

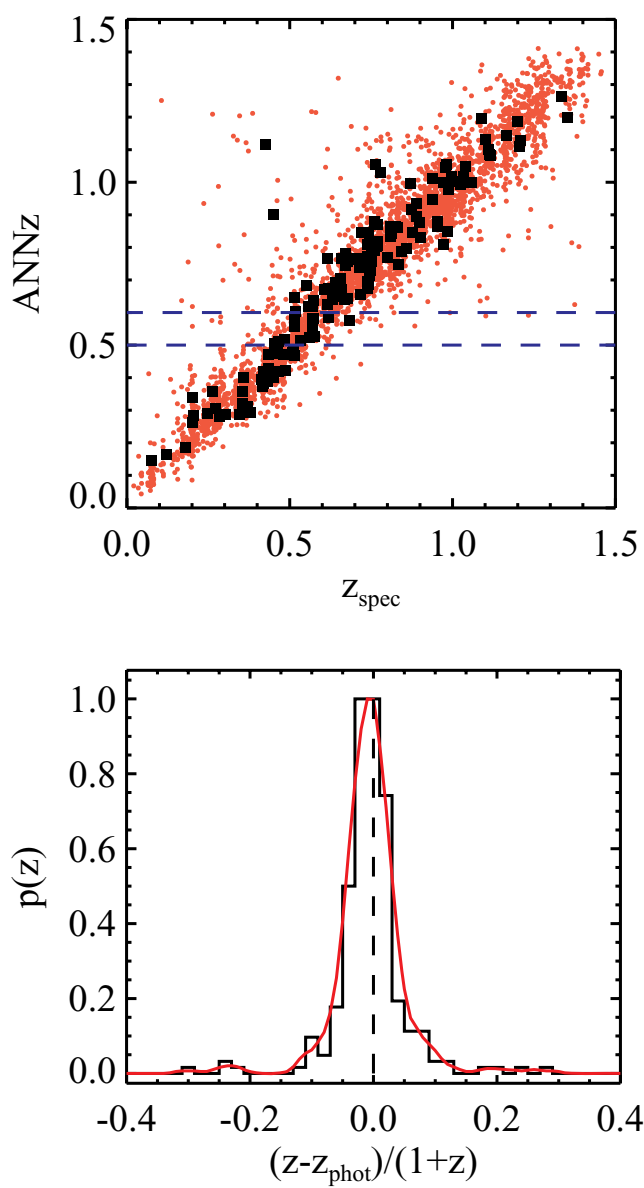

Figure 2. Top panel: comparison of $\mathrm{ANN} z$ and spectroscopic redshifts in AEGIS, shown for all sources (red circles) and X-ray sources (black squares). The $\mathrm{ANN} z$ redshifts generally provide extremely good estimates, although are available for a limited subset of objects, and are restricted to $z<1.5$ where there are sufficient spectroscopic training data. The probability distribution for an $\mathrm{ANN} z$ estimate is determined from the distribution of spectroscopic redshifts for a small slice of photometric redshifts (e.g. blue dashed lines). Bottom panel): the distribution of $\left(z-z_{\text {phot }}\right) /(1+z)$ for the $\mathrm{ANN} z$ redshift range indicated in the top figure. The true distribution (black histogram) is smoothed (red curve) to provide an estimate of probability distribution of true redshifts associated with a given $\mathrm{ANN} z$ estimate.

Neural Network/training set approach (ANNz; Collister \& Lahav 2004). This approach effectively fits an arbitrary function to the redshift-colour relation, using a set of spectroscopic redshifts to train the neural network. As such the redshifts are only expected to be valid for sources with the same range of properties as the training set. The Huang et al. work uses a $3.6 \mu \mathrm{m}$ Infrared Array Camera (IRAC)-selected catalogue (Barmby et al. 2008), further subjected to a number of colour cuts, and utilizes photometry from $M M T$ (Megacam) as well as IRAC, Multiband Imaging Photometer and DEEP2. The neural network was then trained using the DEEP2 spectroscopic data and photometric redshifts estimated for all sources which satisfy the selection criteria. This provides highly accurate photo- $z \mathrm{~s}(\delta z \approx 0.04)$, albeit for a restricted sample. ANN $z$ redshifts are adopted for $38 \mathrm{X}$-ray sources in our sample (without spectroscopic redshifts). To account for the error, we must determine a probability distribution for each redshift. For the ANNz estimates we have based this on the distribution of spectroscopic redshifts, for a given $\mathrm{ANN} z$ estimate, using all objects in the Huang et al. work with spectroscopic identification, as demonstrated in Fig. 2.
For the remaining sources in our sample with secure optical counterparts we adopt photometric redshifts from Ilbert et al. (2006), which are based on the CFHTLS $u^{*} g^{\prime} r^{\prime} i^{\prime} z$ data. The redshifts are based on template fitting and a Bayesian approach, and thus use the same method as the BPZ code. The priors are calibrated using spectroscopic identifications of galaxies in the D1 field of the CFHTLS. The estimates are less reliable than the $\mathrm{ANN} z$ results $(\delta z \approx 0.16)$, but do provide redshift information for the remaining optically identified X-ray sources. However there is a systematic bias for sources with $z_{\text {spec }} \gtrsim 1.2$ to be placed at lower redshifts, as was found for the CDF-S and CDF-N. The authors have made available the full $p(z)$ redshift probability distributions for every source, which we have adopted to account for the uncertainties, though once again will not account for the systematic bias.

\subsubsection{ASCA Large Sky Survey}

The ASCA Large Sky Survey (ALSS; Ueda et al. 1999) covers a contiguous area of $\sim 5 \mathrm{deg}^{2}$ near the North Galactic Pole, and contains 34 sources detected in the hard $(2-7 \mathrm{keV})$ band with the Solid-State Imaging Spectrometer (SIS) detector, down to a flux limit $\sim 10^{-13} \mathrm{erg} \mathrm{s}^{-1} \mathrm{~cm}^{-2}(2-10 \mathrm{keV})$. Optical identifications have been presented by Akiyama et al. (2000). Two of these sources are optically identified as galaxy clusters: one is a star and 30 are associated with AGN, all of which have spectroscopic redshifts. One source remains unidentified. An area curve for this survey was presented by Akiyama et al. (2003), which we adopt for our work. We note that this area curve does not fully account for the Poissonian nature of the X-ray source detection, but as a relatively high flux limit was set for the catalogue and the X-ray fluxes will be accurately measured this is unlikely to have an adverse affect on our bright-end determination.

\subsubsection{ASCA Medium Sensitivity Survey}

The largest area survey in our study is the ASCA Medium Sensitivity Survey (AMSS; Ueda et al. 2001). We include sources from the AMSSn sub-sample, selected in the hard (2-10 keV) band, with optical identifications presented by Akiyama et al. (2003). The sample includes $87 \mathrm{X}$-ray sources, 76 of which are AGN, to a flux limit of $\sim 3 \times 10^{-13} \mathrm{erg} \mathrm{s}^{-1} \mathrm{~cm}^{-2}$, and is 100 per cent optically identified with spectroscopic redshifts available for all the AGN. We include additional sources with redshift identifications from the AMSSs sub-sample (Ueda \& Akiyama, private communication), which includes 20 AGN; two sources in this sample remain unidentified. The area curve for the combined AMSSn and AMSSs samples was presented by Ueda et al. (2003).

\subsection{High-redshift sample}

Due to the catastrophic failure of our photometric redshift estimates at $z \gtrsim 1.2$, we have also investigated an alternative colour preselection approach for identifying AGN at high-redshift, building on the work of Aird et al. (2008). We include four of the fields used in Aird et al. (2008): HDF-N (fully encompassed by the CDF-N X-ray field described above), LALA CETUS, Lynx and EGS1. We additionally include the large area covered by both the AEGIS$\mathrm{X}$ survey and the CFHTLS field D3 optical imaging. This fully encompasses the Groth-Westphal Strip (GWS) field used in Aird et al. (2008), and thus supersedes those data. Our high-redshift data overlap with our hard X-ray-selected samples described in 
Section 2.1; in Section 3.3, we explain how data from our hard $\mathrm{X}$-ray surveys at $z<1.2$ and our high-redshift colour pre-selected samples are combined to determine the evolution of the XLF.

\subsubsection{Optical data}

The reduction of the optical data for HDF-N, LALA CETUS, Lynx and EGS1 and the source detection and photometry with SEXTRACTOR has been described in Aird et al. (2008) and Reddy et al. (2006), and we refer the reader to those papers for further details. For the CFHTLS we have used the publicly released images and catalogues available from Canada Astronomical Data Center, ${ }^{2} 2008$ March release. The catalogues were generated using SExTRACTOR, although with a slightly different configuration to Aird et al. (2008). Initial detection was performed in the $i^{\prime}$ band (which is the deepest band), and magnitudes were determined in the other bands using the Kron (1980) style aperture (determined from the $i^{\prime}$-band data). The data were not smoothed to match the seeing in different bands. These differences will affect the detection of objects and measurements of their colours, although given the depth and quality of the CFHTLS data they are unlikely to adversely affect the colour selection of candidates. Nevertheless, any errors, biases and scatter due to the source detection and photometry procedure will be accounted for by our simulations to derive the optical selection functions (Section 3.3.1).

Samples of $z \sim 3$ Lyman-break galaxies (LBGs) were extracted from the optical catalogues using the standard selection criteria from Steidel et al. (2003):

$\mathrm{G}-\mathcal{R} \leq 1.2$

$U_{n}-\mathrm{G} \geq \mathrm{G}-\mathcal{R}+1.0$

$19.0 \leq \mathcal{R} \leq 25.5$

with the faint limit of $\mathcal{R} \leq 25.0$ for EGS1. An additional sample of objects at slightly lower redshifts is selected using the $\mathrm{BX}$ selection criteria (Adelberger et al. 2004):

$\mathrm{G}-\mathcal{R} \geq-0.2$

$U_{n}-\mathrm{G} \geq \mathrm{G}-\mathcal{R}+0.2$

$\mathrm{G}-\mathcal{R} \leq 0.2\left(U_{n}-\mathrm{G}\right)+0.4$

$U_{n}-\mathrm{G}<\mathrm{G}-\mathcal{R}+1.0$.

These objects are 'sub-U dropouts', falling in a thin slice of colour space below the Lyman-break criteria, with a selection function that peaks around $z \sim 2.5$ (see Section 3.3.1).

Objects were selected from the CFHTLS optical data using the same criteria. However, there are significant differences between the CFHTLS filter set and our $U_{n}$ GR filters used in the other fields. The $\mathcal{R}$ band falls between the $r^{\prime}$ and $i^{\prime}$ filters; thus, the total $\mathcal{R}$-band magnitudes were calculated for every object using an approximate colour correction, given by

$\mathcal{R}=r^{\prime}+0.05-1.08\left(r^{\prime}-i^{\prime}\right)$,

which was determined from our model colour distributions described in Section 3.3.1. These estimated $\mathcal{R}$ magnitudes were used to apply the total magnitude limits, consistent with our other fields. However, for the selection in colour space we used the original $u^{*} g^{\prime} r^{\prime}$ magnitudes in place of $U_{n} \mathrm{G} \mathcal{R}$, thus avoiding the effects

\footnotetext{
${ }^{2}$ http://www4.cadc-ccda.hia-iha.nrc-cnrc.gc.ca/community/CFHTLS-SG/ docs/cfhtls.html
}

of uncertain colour corrections. The differences in the filters will lead to different selection functions, which we determine in Section 3.3.1.

\subsubsection{X-ray data}

All of our fields have been observed with Chandra, and have exposure times of $\gtrsim 200 \mathrm{ks}$. The data have been reduced using our pipeline procedure (Laird et al. 2009). For our high-redshift samples we use sources detected in the soft band with a significance of $<4 \times 10^{-6}$. Our colour pre-selection limits us to sources at $z \gtrsim$ 2 , and at these redshifts the soft band will probe rest-frame energies of $\sim 2-8 \mathrm{keV}$. Thus our selection should be relatively unbiased for the selection of moderately absorbed $\operatorname{AGN}\left(N_{\mathrm{H}} \lesssim 10^{23} \mathrm{~cm}^{-2}\right)$, and is comparable with the hard-band selection employed at lower redshifts (Cowie et al. 2003; Barger et al. 2005).

\subsubsection{Matching the $X$-ray and optical samples}

We determined which of our colour pre-selected high-redshift objects host AGN by matching the optical and X-ray source lists. The matching is performed with the entire $\mathcal{R}$ - (or $i^{\prime}$-) selected optical catalogues using the likelihood ratio method. The colour selection criteria are then applied to the matches. Matching the entire optical catalogue ensures that X-ray sources are matched to the most likely optical counterparts and prevents colour pre-selected objects being erroneously associated with nearby bright X-ray sources, which have correspondingly bright, secure optical counterparts (cf. the direct extraction method used in Aird et al. 2008).

In Table 2, we list the total numbers of X-ray-detected objects within each of our colour-selected samples. We searched for spectroscopic redshifts for our sources as described in Sections 2.1.2 and 2.1.3 for the HDF-N and AEGIS-X fields respectively, and from Stern et al. (2002) for the Lynx field. The remaining unidentified sources were assigned redshift probability distributions, $p(z)$, based on the optical selection functions (see Section 3.3.1).

\subsubsection{Low-redshift interlopers}

$U_{n}$ GR LBG selection at $z \sim 3$ is known to be extremely clean and contains very few low-redshift interlopers (Steidel et al. 2003). The majority of interlopers are Galactic stars, which are not expected to be bright X-ray sources and thus will not contaminate our sample. However, the BX selection, which does not rely on very strong features such as the Lyman break to identify candidates, is more susceptible to contamination from low-redshift sources, mainly from low-redshift $(z \lesssim 0.3)$ star-forming galaxies in which the Balmer break is confused with the Lyman $\alpha$ forest absorption at $z \gtrsim 2$ (Adelberger et al. 2004; Reddy et al. 2006). Indeed, a significant number of low-redshift $(z<1.2)$ interlopers are identified within our spectroscopically observed, X-ray-detected BX sample (see Table 2 and Fig. 3). By examining the positions of these sources in the $f_{\mathrm{X}}-f_{\text {opt }}$ plane (see Fig. 4) it is clear that five of these low-redshift interlopers occupy a distinct position, associated with optically bright objects $(\mathcal{R}<23)$, but with relatively low X-ray fluxes; thus, $\log \left(f_{\mathrm{X}} / f_{\text {opt }}\right)<-1.5$. These objects are the expected bright, low-redshift star-forming galaxies, with X-ray emission associated with star formation processes. We exclude two more X-ray-detected BX sources from our analysis which fall in the same region and are thus also suspected to be low-redshift contaminants. The faint $\mathrm{BX}$ source with $\mathcal{R} \approx 23.5$ and 
Table 2. Summary of the fields used to determine the high-redshift XLF using rest-frame UV colour pre-selection.

\begin{tabular}{|c|c|c|c|c|c|c|c|c|c|c|c|}
\hline $\begin{array}{l}\text { Field } \\
\text { name }\end{array}$ & $\begin{array}{c}\text { RA } \\
(\mathrm{J} 2000)\end{array}$ & $\begin{array}{c}\text { Dec } \\
(\mathrm{J} 2000)\end{array}$ & $\begin{array}{c}N_{\mathrm{H}}{ }^{a} \\
\left(10^{20} \mathrm{~cm}^{-2}\right)\end{array}$ & $\begin{array}{c}\text { X-ray } \\
\text { exposure }^{b} \\
(\mathrm{ks})\end{array}$ & $\begin{array}{c}\text { Survey } \\
\operatorname{area}^{c} \\
\left(\operatorname{arcmin}^{2}\right)\end{array}$ & Selection & $N_{\text {cand }}{ }^{d}$ & $N_{\mathrm{X}}{ }^{e}$ & $N_{\text {spec }^{f}}^{f}$ & $\begin{array}{l}N_{\text {low } z^{g}} \\
z<1.4\end{array}$ & $N_{\text {samp }}{ }^{h}$ \\
\hline HDF-N & $12: 36: 55$ & $+62: 14: 18$ & 1.5 & 1862.9 & 149.1 & LBG & 292 & 3 & 2 & 0 & 3 \\
\hline \multirow[t]{2}{*}{ AEGIS-X/CFHTLS } & \multirow[t]{2}{*}{$14: 17: 43$} & \multirow[t]{2}{*}{$+52: 28: 25$} & \multirow[t]{2}{*}{1.2} & \multirow[t]{2}{*}{188.7} & \multirow[t]{2}{*}{1155.6} & $\mathrm{BX}$ & 17050 & 77 & 26 & 10 & 66 \\
\hline & & & & & & LBG & 6933 & 30 & 10 & 0 & 30 \\
\hline \multirow[t]{2}{*}{ EGS1 } & \multirow[t]{2}{*}{$14: 22: 43$} & \multirow{2}{*}{$+53: 25: 25$} & \multirow[t]{2}{*}{1.2} & \multirow[t]{2}{*}{177.8} & \multirow[t]{2}{*}{358.9} & $\mathrm{BX}$ & 809 & 8 & 0 & 0 & 7 \\
\hline & & & & & & LBG & 329 & 7 & 0 & 0 & 7 \\
\hline \multirow[t]{2}{*}{ LALA CETUS } & \multirow[t]{2}{*}{ 02:04:44 } & \multirow[t]{2}{*}{$-05: 05: 34$} & \multirow[t]{2}{*}{2.2} & \multirow[t]{2}{*}{173.1} & \multirow[t]{2}{*}{233.3} & $\mathrm{BX}$ & 660 & 4 & 0 & 0 & 4 \\
\hline & & & & & & LBG & 144 & 3 & 0 & 0 & 3 \\
\hline
\end{tabular}

${ }^{a}$ Galactic column density from Dickey \& Lockman (1990); ${ }^{b}$ X-ray exposure time after good time interval and background filtering (average of the individual pointings for AEGIS-X); ${ }^{c}$ total survey area covered by X-ray and optical data; ${ }^{d}$ number of candidates from the optical imaging satisfying the colour selection criteria; ${ }^{e}$ number of the colour-selected candidates with secure X-ray counterparts; ${ }^{f}$ number of the X-ray-detected candidates with spectroscopic redshifts; ${ }^{g}$ number of the spectroscopically identified sample which are low-redshift interlopers $(z<1.4) ;{ }^{h}$ final number of sources in our sample after excluding spectroscopically identified low-redshift interlopers and additional suspected low-redshift interlopers according to their position in the $f_{\mathrm{X}}-f_{\text {opt }}$ plane (see Section 2.2.4, Fig. 4).

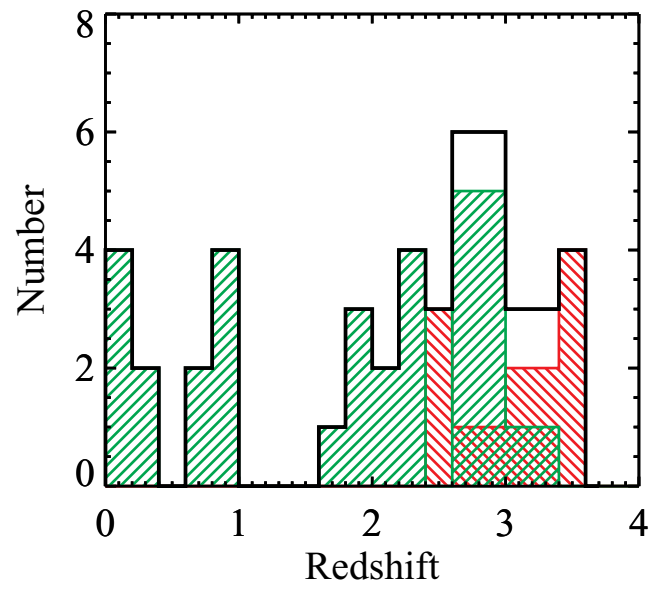

Figure 3. Distribution of available spectroscopic redshifts for the X-raydetected colour-selected objects. Green: BX, red: LBG and black: total. The BX sample is contaminated by low-redshift interlopers, although around half may be identified as low-redshift star-forming galaxies and on the basis of their $f_{\mathrm{X}} / f_{\text {opt }}$ ratio (see Fig. 4, Section 2.2.4).

$\log \left(f_{\mathrm{X}} / f_{\text {opt }}\right)<-1.5$, which has a spectroscopic redshift in the correct range, is also excluded as we suspect a starburst origin (see also Aird et al. 2008).

The six remaining interlopers, however, are associated with low-redshift AGN, and thus fall within the AGN region $-1.0 \lesssim$ $\log \left(f_{\mathrm{X}} / f_{\text {opt }}\right) \lesssim+1.0$. While any AGN which have been spectroscopically identified as low-redshift interlopers are excluded from our analysis, additional contaminants may remain within our unidentified BX sample. Based on our spectroscopic sample the fraction of low-redshift AGN contaminants may be as high as $\sim 25$ per cent, although our sample is biased in the sense that a higher fraction of bright objects has spectroscopic identifications. Indeed, in the large sample of BX-selected galaxies with spectroscopic redshifts used by Reddy et al. (2008) the low-redshift contamination in the galaxy sample was $\sim 59$ per cent for bright candidates $(\mathcal{R}<23.5)$, whereas for fainter candidates it was only $\sim 9$ per cent. Conversely, in the sample of objects spectroscopically identified as

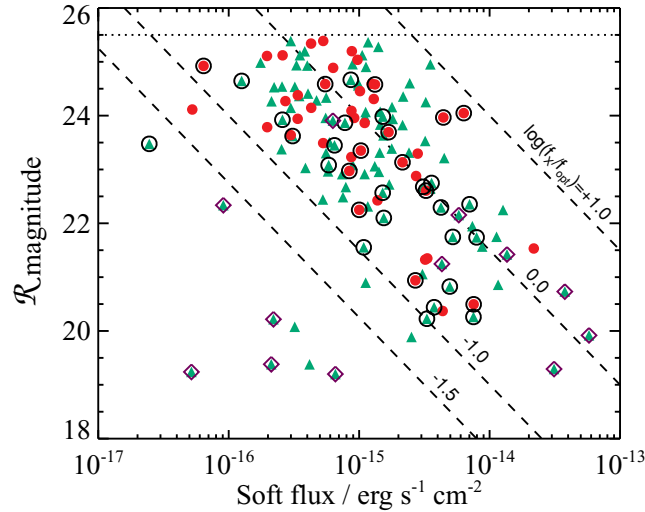

Figure 4. X-ray flux $(0.5-2 \mathrm{keV})$ versus $\mathcal{R}$ magnitude for the $\mathrm{BX}$ (green triangles) and LBG (red circles) X-ray-detected samples. Black circles indicate objects with spectroscopic redshifts, $z_{\text {spec }}>1.4$; purple diamonds indicate low-redshift interlopers with $z_{\text {spec }}<1$.4. As discussed in Section 2.2.4 a number of the interlopers are associated with low-redshift starforming galaxies, which are optically bright but very faint in X-rays. We thus exclude all sources with $\log \left(f_{\mathrm{X}} / f_{\text {opt }}\right)<-1.5$. This will exclude the low-redshift interlopers, in addition to bright high-redshift starburst galaxies that may also contaminate the AGN XLF at the faintest fluxes (see Aird et al. 2008). Objects with $z_{\text {spec }}<1.4$ are also excluded from our analysis, but interlopers associated with low- $z$ AGN may still contaminate the remaining spectroscopically unidentified BX-selected sample.

AGN (including QSOs and narrow-line AGN), the contamination fraction was only $\sim 12$ per cent and approximately consistent over the entire magnitude range. Clearly a much larger, near complete sample of spectroscopic redshifts for BX/X-ray-selected AGN is required to truly determine the interloper fraction within our sample, and could also allow improvements to the selection criteria (such as introducing data from redder wavebands) to be developed to help reduce the contamination. However, given the current uncertainties, we have chosen not to make further corrections to account for the interloper fraction within our sample. We thus note that the faint end of our XLF based on the BX sample may be overestimated due to low-redshift contamination. 


\subsubsection{High-luminosity sample}

The total area coverage for our high-redshift sample remains relatively small, and thus we require an additional sample of highluminosity AGN to constrain the bright end of the XLF. We have chosen to use bright objects $\left(f_{\mathrm{X}}>5 \times 10^{-15} \mathrm{erg} \mathrm{s}^{-1} \mathrm{~cm}^{-2}\right)$ from the Hasinger, Miyaji \& Schmidt (2005) compilation of surveys (excluding the CDF-N to avoid overlap with our own analysis). These samples provide a soft-band selected, highly complete, spectroscopically confirmed sample. We have included objects with $1.9<z \leq$ 3.5 , providing 30 high-luminosity AGN in our high-redshift sample. These bright, complete, spectroscopic surveys may be combined with our colour pre-selected samples by assuming a top-hat selection function over the chosen redshift range (see Section 3.3.2).

\section{A BAYESIAN APPROACH TO DETERMINING THE XLF}

Our compilation of X-ray surveys provides large samples of AGN over a range of redshifts with well-defined completeness, required to determine the evolution of the XLF. However, the faint sources from the Chandra fields are subject to uncertainty in both the redshift determination (for those sources with photometric redshifts or in the high- $z$ colour pre-selected samples) and the X-ray flux measurement (due to the small numbers of counts from the faintest sources). This has led us to develop a Bayesian methodology to make accurate inferences about the form and evolution of the XLF, accounting for the uncertainties in individual sources.

\subsection{The likelihood function}

The first step is to determine the likelihood function, $\mathcal{L}$, for which we have followed the work of Loredo (2004). Our individual sources are effectively Poissonian points, drawn from the distribution given by the XLF. Thus, for a given model XLF, the expected number of detectable sources, $\lambda$, is given by

$\lambda=\int \mathrm{d} \log L_{\mathrm{X}} \int \frac{\mathrm{d} V}{\mathrm{~d} z} \mathrm{~d} z A\left(L_{\mathrm{X}}, z\right) \phi\left(L_{\mathrm{X}}, z \mid \theta\right)$,

where the integrals are taken over the entire range of possible values. $A\left(L_{\mathrm{X}}, z\right)$ is the area of the survey sensitive to a source of a particular luminosity, $L_{X}$, and redshift, $z$, and thus a particular flux if we assume a single spectral shape (power law with a photon index of $\Gamma=1.9)$. $A\left(L_{\mathrm{X}}, z\right)$ effectively describes the probability of a source being detected. The luminosity function, $\phi\left(L_{\mathrm{X}}, z \mid \theta\right)$, is the differential number density of sources per unit comoving volume as a function of $L_{\mathrm{X}}$ and $z$, given an assumed model described by the set of parameters, $\theta \cdot \frac{\mathrm{d} V}{\mathrm{~d} z}$ is the differential comoving volume per unit area, as a function of $z$.

As described in Loredo (2004), the likelihood function may be constructed from the product of the probabilities of the observed data from the individual sources, given the model, and the probability that no other objects were detected. For a Poisson process, the probability of no objects being detected is simply $\mathrm{e}^{-\lambda}$. In the presence of individual source uncertainties, the probability of the observed data, $d_{i}$, from an individual source $i$, given the model XLF is found by marginalizing $p\left(d_{i} \mid L_{\mathrm{X}}, z\right)$ over the model distribution of $L_{\mathrm{X}}$ and $z$. Thus,

$$
\begin{aligned}
& \mathcal{L}\left(\left\{d_{i}\right\} \mid \theta\right)= \\
& \mathrm{e}^{-\lambda} \times \prod_{i=1}^{M} \int \mathrm{d} \log L_{\mathrm{X}} \int \frac{\mathrm{d} V}{\mathrm{~d} z} \mathrm{~d} z p\left(d_{i} \mid L_{\mathrm{X}}, z\right) \phi\left(L_{\mathrm{X}}, z \mid \theta\right),
\end{aligned}
$$

where $\left\{d_{i}\right\}$ represents the set of observed data from all sources, and the product in the second part of the equation is over $M$-detected sources. For the idealized situation where the redshift and luminosity are perfectly determined, $p\left(d_{i} \mid L_{\mathrm{X}}, z\right)$ will be a delta function at the true values, and equation (6) will generalize to the standard maximum likelihood estimator. For our sample $p\left(d_{i} \mid L_{\mathrm{X}}, z\right)$ is the convolution of the redshift probability distribution $p(z)$ and the Poisson likelihood of obtaining the observed X-ray data, $N$ counts:

$\mathcal{L}(N \mid s, b)=\frac{(s+b)^{N}}{N !} \mathrm{e}^{-(s+b)}$,

where $s$ is calculated from the hard-band $(2-10 \mathrm{keV})$ flux for a source of luminosity $L_{\mathrm{X}}$ and redshift $z$, assuming $\Gamma=1.9$ and Galactic $N_{\mathrm{H}}$. Thus, the Poissonian nature of the counts from the X-ray sources, the associated uncertainty in the flux and the Eddington bias are naturally accounted for in our determination of the XLF. Assuming $\Gamma=1.9$ approximately corrects for absorption effects due to intrinsic column densities $N_{\mathrm{H}} \lesssim 10^{23} \mathrm{~cm}^{-2}$ at $z \sim 1$, although we have not chosen to make a more sophisticated correction on a source-by-source basis (see further discussion in Section 5.2). Our approach builds on the $\log N-\log S$ work of Georgakakis et al. (2008), extended to include redshift information, and allows us to use the improved method of calculating the X-ray sensitivity. For sources with spectroscopic redshifts, we assume delta functions for $p(z)$. We also assume delta functions for the luminosities of sources in both the hard and soft X-ray-selected, large area, bright samples (Sections 2.1.4, 2.1.5 and 2.2.5).

\subsection{Correcting for incompleteness}

The above likelihood function does not account for the incompleteness of our samples. In our hard X-ray-selected samples, between $\sim 14$ per cent (AEGIS) and $\sim 32$ per cent (CDF-N, CDF-S) of the sources lack secure optical counterparts, and thus have neither spectroscopic nor photometric redshifts. However, we can derive a completeness correction term to account for the incompleteness in the likelihood function.

Fig. 5(a) shows the distribution of the hard X-ray flux and optical magnitudes of our (optically identified) sources. Sources are scattered over a range of optical to X-ray flux ratios, approximately over the range $-1<\log f_{\mathrm{X}} / f_{\text {opt }}<1$ although a small proportion is at lower ratios and a significant number of this hard X-ray-selected sample exhibit $\log f_{\mathrm{X}} / f_{\text {opt }}>1$. At faint $\mathrm{X}$-ray fluxes, a large fraction will be unidentified due to their faint optical magnitudes. In Fig. 5(b), we show the $\log f_{\mathrm{X}} / f_{\text {opt }}$ distribution of our sources. A Gaussian approximation, taking the sample mean, $\mu$, and standard deviation, $\sigma$, is shown in red, and provides a reasonable description of this distribution. If we apply a strict magnitude limit of $R=25.5$ (sources fainter than this limit are now deemed unidentified), and assume that all the unidentified sources would have optical counterparts fainter than this limit (reasonable given the very deep optical data we have used to search for counterparts), then we can introduce a correction to the expected number of sources, $\lambda$, given by equation (4), based on the fraction of the $\log f_{\mathrm{X}} / f_{\text {opt }}$ distribution probed at a given flux. Thus,

$\lambda$

$=\int \mathrm{d} \log L_{\mathrm{X}} \int \frac{\mathrm{d} V}{\mathrm{~d} z} \mathrm{~d} z \int \mathrm{d} R A\left(L_{\mathrm{X}}, z\right) \phi\left(L_{\mathrm{X}}, z \mid \theta\right) g\left(L_{\mathrm{X}}, z, R \mid \mu, \sigma\right)$

$=\int \mathrm{d} \log L_{\mathrm{X}} \int \frac{\mathrm{d} V}{\mathrm{~d} z} \mathrm{~d} z A\left(L_{\mathrm{X}}, z\right) \phi\left(L_{\mathrm{X}}, z \mid \theta\right) C\left(L_{\mathrm{X}}, z \mid \mu, \sigma\right)$, 

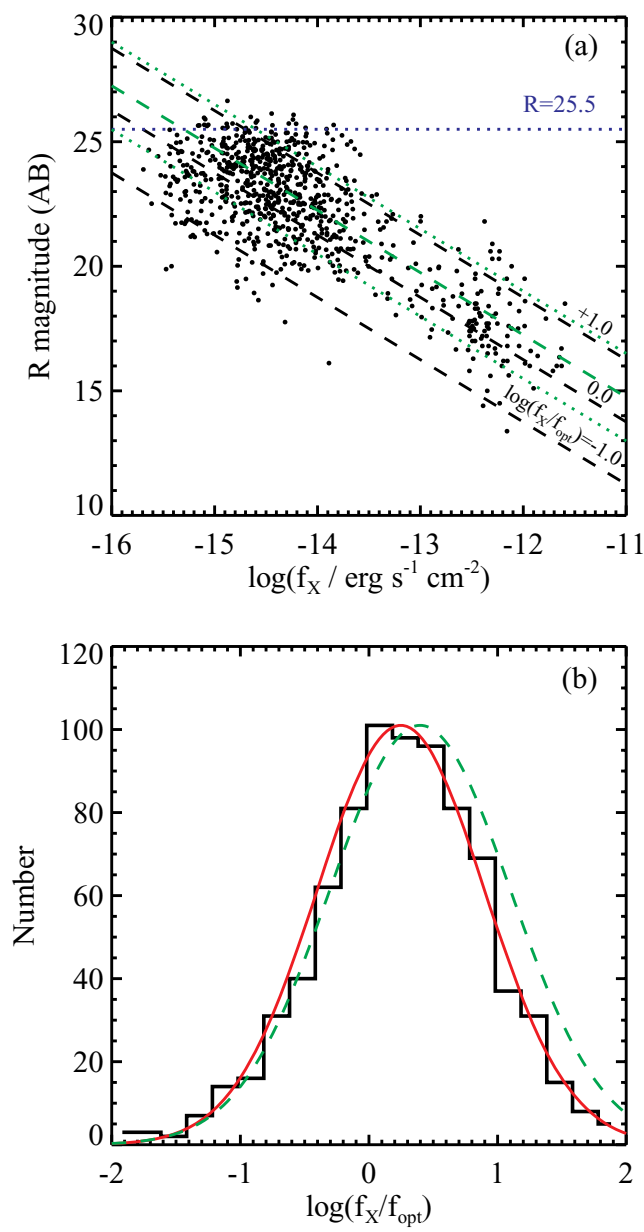

Figure 5. (a) Hard (2-10 keV) flux against $R$ magnitude (AB) for our entire hard X-ray-selected sample (black circles). Black dashed lines: $\log f_{\mathrm{X}} / f_{\text {opt }}=-1.0,0.0$ and +1.0 , indicating the typical range of AGN. Green lines: mean (dashed) and $\pm 1 \sigma$ (dotted) of the best-fitting Gaussian $\log f_{\mathrm{X}} / f_{\text {opt }}$ distribution (see Section 4.2). Blue dotted line: $R=25.5$ limit for including optical counterparts. (b) Histogram of the distribution of $\log f_{\mathrm{X}} / f_{\text {opt }}$ (black) and the Gaussian distribution corresponding to the sample mean and standard deviation (red curve). This distribution will be biased due to incomplete optical identifications, as well as the distribution of X-ray fluxes. We account for these effects when fitting our evolutionary models (see Sections 3.2 and 4) and derive the $\log f_{\mathrm{X}} / f_{\text {opt }}$ distribution (green dashed curve), which is shifted to higher $f_{\mathrm{X}} / f_{\text {opt }}$ ratios.

where $g\left(L_{\mathrm{X}}, z, R \mid \mu, \sigma\right)$ describes the $R$-magnitude distribution of sources at a given flux, $f_{\mathrm{X}}\left(L_{\mathrm{X}}, z\right)$, and is derived from the Gaussian distribution of $\log f_{\mathrm{X}} / f_{\text {opt }} \cdot g\left(L_{\mathrm{X}}, z, R \mid \mu, \sigma\right)$ is normalized such that

$\int_{-\infty}^{\infty} \mathrm{d} R g\left(L_{\mathrm{X}}, z, R \mid \mu, \sigma\right)=1$.

Thus, the factor

$C\left(L_{\mathrm{X}}, z \mid \mu, \sigma\right)=\int_{-\infty}^{25.5} \mathrm{~d} R g\left(L_{\mathrm{X}}, z, R \mid \mu, \sigma\right)$

is a completeness correction, reducing the expected number of detectable sources with low X-ray fluxes.

However, the observed distribution (the red curve in Fig. 5) will be biased by the very incompleteness we are trying to correct for, as sources with $R \gtrsim 25.5$ will be missed. The extent of the bias will depend on the flux distribution of the X-ray sources (and thus the $\mathrm{XLF}$, and the X-ray sensitivity), which determines the number of sources with faint $\mathrm{X}$-ray fluxes where the problem arises. Therefore, to improve our completeness correction the $\log f_{\mathrm{X}} / f_{\text {opt }}$ distribution should be determined simultaneously with the XLF, accounting for the incompleteness, using the observed data (the $R$-magnitudes of the detected sources). We therefore introduce $g\left(L_{\mathrm{X}}, z, R\right)$ into the second term in equation (6), modifying the likelihood to

$$
\begin{aligned}
& \mathcal{L}\left(\left\{d_{i}\right\} \mid \theta, \mu, \sigma\right)=\mathrm{e}^{-\lambda} \prod_{i=1}^{M} \int \mathrm{d} \log L_{\mathrm{X}} \\
& \times \int \frac{\mathrm{d} V}{\mathrm{~d} z} \mathrm{~d} z p\left(d_{i} \mid L_{\mathrm{X}}, z\right) \phi\left(L_{\mathrm{X}}, z \mid \theta\right) g\left(L_{\mathrm{X}}, z, R_{i} \mid \mu, \sigma\right),
\end{aligned}
$$

where we neglect any uncertainty in the measurement of the $R$ magnitude of an individual source, $R_{i}$, which is a minor simplification. The parameters $\mu$ and $\sigma$ are introduced as nuisance parameters, and will be determined along with the XLF, integrating over their uncertainties when making inferences regarding the XLF parameters, $\theta$. In Fig. 5(b), the green dashed line indicates the best-fitting (mode) values of $\mu=0.24 \pm 0.01$ and $\sigma=0.68 \pm 0.01$ for our luminosity and density evolution (LADE) evolutionary model (Section 4.2). The peak of the distribution is clearly shifted to a higher $f_{\mathrm{X}} / f_{\text {opt }}$, indicating the bias in the simple estimates.

\subsection{Incorporating the high- $z$ colour pre-selected samples}

In Section 4, we present results for the evolution of the XLF over the full available redshift range based on our hard X-ray-selected sample only (using spectroscopic and photometric redshifts). However, due to the systematic bias in photo- $z$ estimates at $z \gtrsim 1.2$, we also wish to derive the evolution using our high-redshift colour pre-selected samples. Due to the a priori colour selection, these samples will suffer from large, but well-defined, incompleteness. Accounting for this incompleteness and combining our soft-bandselected high- $z$ sample with the hard X-ray-selected samples at lower redshifts require a number of further modifications to our methodology.

\subsubsection{Optical selection functions}

The first step is to determine the optical selection functions for the colour selection criteria. These were determined using the simulation method described in Aird et al. (2008) (see also Steidel et al. 1999; Hunt et al. 2004; Reddy et al. 2008). Three sets of template spectra were determined for different spectroscopic classifications (QSO, NLAGN or GAL; see Steidel et al. 2003), and used to generate model $U_{n}$ GR and $u^{*} g^{\prime} r^{\prime}$ colour distributions. GAL and NLAGN colours were generated using a Bruzual \& Charlot (2003) model template spectrum for a galaxy with continuous star formation and an age of $1 \mathrm{Gyr}$. This template spectrum was reddened using the Calzetti et al. (2000) relation for obscuration by dust, with various extinction coefficients drawn from the distributions based on the observed range of UV spectral slopes for GAL and NLAGN sources in LBG samples (Adelberger \& Steidel 2000; Steidel et al. 2002, 2003). Such modelling is not a full physical description for the NLAGN, being based only on a template spectrum for star-forming processes, but is sufficient to simulate the observed range of spectral shapes and redder distribution of broadband colours for NLAGN. For QSOs, we have followed the work of Hunt et al. (2004). The template spectra were generated by varying the continuum slope and $\mathrm{Ly} \alpha$-equivalent widths of a composite of 59 QSO spectra (corrected for intergalactic absorption) from Sargent, Steidel \& Boksenberg (1989), based on the Gaussian distributions given in Hunt et al. (2004). These model spectra for all three types 

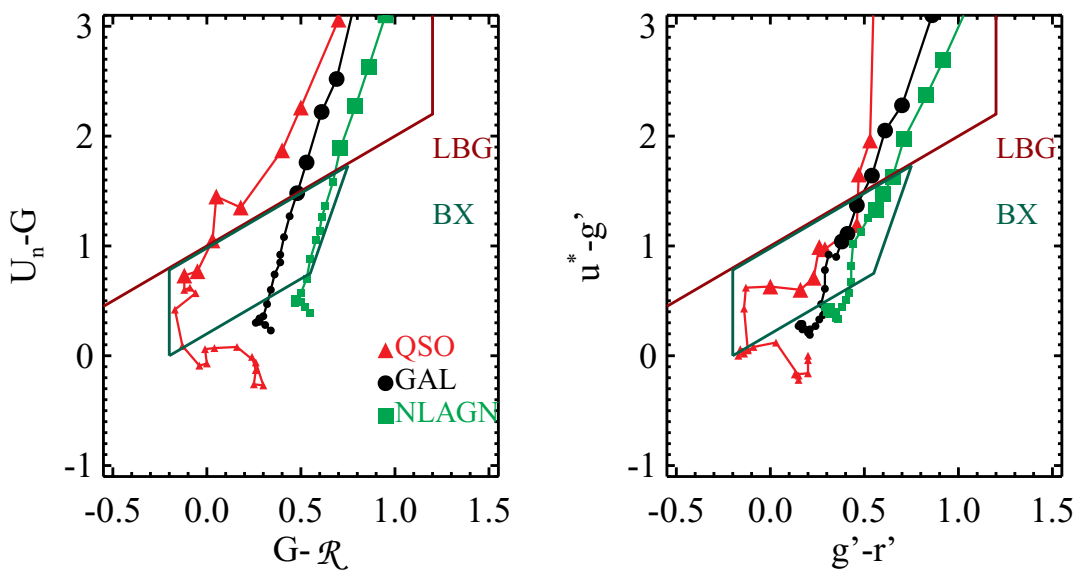

Figure 6. Example model colour distributions for our GAL (black circles), NLAGN (green squares) and QSO (red triangles) classifications. The same model for each type is shown in $U_{n} \mathrm{GR}$ (left-hand panel) and $u^{*} g^{\prime} r^{\prime}$ (right-hand panel) colour-space. The model is redshifted over $1.0<z<4.0$. Marks show the colours at $\Delta z=0.1$ intervals, with larger symbols indicating $2.5<z<3.5$. The variation between colours through different filter sets leads to differences in the optical selection functions (see Fig. 7, Section 3.3.1).

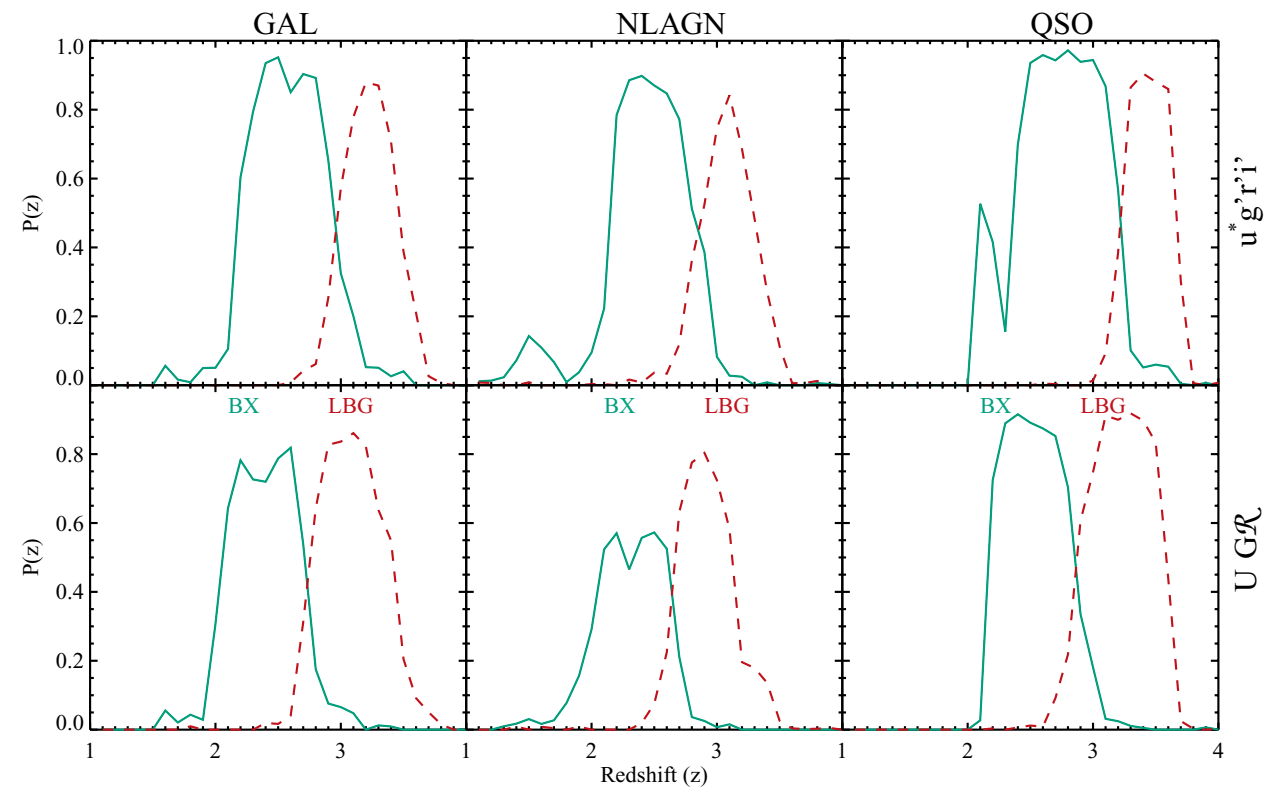

Figure 7. Optical selection functions for our BX (green solid lines) and LBG (red dashed lines) colour selection criteria. The top row shows the selection functions for the CFHTLS-D3 optical data and $u^{*} g^{\prime} r^{\prime} i^{\prime}$ filter sets. The bottom row shows selection functions in the HDF-N (with optical data of comparable depth) using $U_{n} \mathrm{G} \mathcal{R}$ filters. Each column is for a different spectroscopic classification as indicated. Significant differences in efficiency and redshift coverage exist between the selection functions for different optical types and filter sets (see discussion in Section 3.3.1).

were then redshifted over the range $z=1-4$ and intergalactic absorption was applied by simulating a random line of sight to each object with intervening Lyman-limit absorption systems distributed according to the MC-NH model of Bershady, Charlton \& Geoffroy (1999), as described by Hunt et al. (2004). The final spectra are multiplied by the filter transmission curves to produce the model colour distributions. Fig. 6 compares colours for a single model spectrum (for each classification) at different redshifts in both $U_{n}$ $\mathrm{G} \mathcal{R}$ and $u^{*} g^{\prime} r^{\prime}$ colour space.

The full colour distributions were used to add artificial objects to the optical imaging data. Selection functions are then determined as the probability of recovering an object with a given $\mathcal{R}$ magnitude with either the LBG or BX selection as a function of redshift. Objects were recovered using our SEXTRACTOR routines (see Aird et al. 2008) in the $U_{n} \mathrm{G} \mathcal{R}$ fields. The SExTRACTOR configuration file used to generate the released catalogues for CFHTLS is available on-line; ${ }^{3}$ thus, we used this configuration for those data. Objects were selected in the $i^{\prime}$-band image, and flexible Kron (1980) style apertures were used to measure the magnitudes in other bands and calculate the output colours. Our selection functions will thus account for the different source detection and photometry technique, and the effects of the different filter sets.

Fig. 7 compares our final selection functions (derived from the simulations) for the different colour criteria, filter sets and optical classifications. There are clear differences between the $U_{n} \mathrm{G} \mathcal{R}$ and $u^{*} g^{\prime} r^{\prime}$ selections, which we expect given the varying wavelength

\footnotetext{
${ }^{3}$ http://www1.cadc-ccda.hia-iha.nrc-cnrc.gc.ca/community/CFHTLS-SG/ docs/cfhtlscat.sex
} 
coverage of the filter sets, and results in variation in our selection functions. The $u^{*}$ filter extends to longer wavelengths than the $U_{n}$ filter, and as such the $u^{*}-g^{\prime}$ colour becomes sensitive to the Lyman break at higher redshifts than the $U_{n}-\mathrm{G}$ colour, as can be seen from the tracks in Fig. 6. Thus the LBG selection functions are shifted to slightly higher redshifts when using $u^{*} g^{\prime} r^{\prime}$ rather than the $U_{n} \mathrm{G} \mathcal{R}$ filter sets. The same effect leads to BX selection functions which probe to higher redshifts, although the low-redshift cut-off is relatively unaffected. Thus, the BX selection functions are generally broader for $u^{*} g^{\prime} r^{\prime}$ selection.

The red continuum of our NLAGN model generally leads to a reduced efficiency of the selection functions. This effect is particularly seen for the BX selection function for $U_{n} \mathrm{G} \mathcal{R}$ colours. The example NLAGN model shown in Fig. 6 has colours which are very close to the boundary of the BX selection box. The intrinsic scatter we apply to our models and the photometric scatter introduced via our simulations will both lead to incompleteness of the BX selection function, which is clearly seen in Fig. 7 (bottom centre panel). However, the $r^{\prime}$ filter is significantly bluer than the $\mathcal{R}$ bandpass, resulting in $g^{\prime}-r^{\prime}$ colours which are generally bluer than $\mathrm{G}-\mathcal{R}$, and thus the NLAGN BX selection function is less adversely affected by the red continuum for $u^{*} g^{\prime} r^{\prime}$ selection, and has a higher efficiency (cf. top and bottom central panels in Fig. 7). The same effect is seen for the GAL BX selection functions, although it is much milder as the distribution of GAL colours is not as red as the distribution of NLAGN colours.

The model tracks for the QSO colours in Fig. 6 follow complicated routes which have very different shapes for the different filter sets. This is due to the presence of the strong, broad emission lines, which will move in and out of the various filters at different redshifts. However, the basic characteristics necessary for our colour selections remain present: the Lyman break leads to a rapid reddening of the $U_{n}-\mathrm{G}$ ( or $u^{*}-g^{\prime}$ ) colour at $z \sim 3$ and a characteristically flat spectrum is found at lower redshifts.

\subsubsection{Modifying the likelihood function}

The optical selection functions described above can be used to modify the likelihood function derived in Section 3.2, to allow us to combine the hard X-ray-selected samples at low redshifts $(z<1.2)$ and our colour pre-selected high-redshift samples. First, $A\left(L_{X}, z\right)$, which effectively gives the probability of detecting a source with given $L_{\mathrm{X}}$ and $z$ must be modified to account for the two different selection techniques. For $z<1.2, A\left(L_{\mathrm{X}}, z\right)$ is calculated from the area curves for our hard X-ray surveys, converting the hard (observed 2-10 keV) X-ray flux to an $L_{\mathrm{X}}$ (rest-frame $2-10 \mathrm{keV}$ ) and $z$ assuming $\Gamma=1.9$ and Galactic $N_{\mathrm{H}}$, as before. For $z>1.2$, we use the area as a function of the soft X-ray flux, multiplied by the average of the combined BX and LBG selection functions, $\tilde{P}_{f}(z)$, for all magnitudes and spectroscopic classifications for a field, scaled to a maximum of 1 . Thus,

$\tilde{P}_{f}(z) \propto \sum_{\text {types }} \int_{19.0}^{25.5} P_{f}(\mathcal{R}, z) \mathrm{d} \mathcal{R}$

$A\left(L_{\mathrm{X}}, z\right)=\sum_{\text {fields }} A_{\mathrm{f}}\left(f_{\mathrm{X}}\left(L_{\mathrm{X}}, z\right)\right) \times \tilde{P}_{f}(z)[z>1.2]$,

where $A_{\mathrm{f}}\left(f_{\mathrm{X}}\left(L_{\mathrm{X}}, z\right)\right)$ is the area of each field sensitive to a soft $(0.5-$ $2 \mathrm{keV}$ ) flux for a given $2-10 \mathrm{keV}$ luminosity source at $z$, assuming $\Gamma=1.9$ and Galactic $N_{\mathrm{H}}$, for each field. This reduces the probability of objects being detected outside of the range covered by the colour pre-selection, and thus provides realistic expected numbers at high redshifts in the likelihood evaluation (equation 10). As we consider the uncertainty in the X-ray flux, we can utilize the method of Georgakakis et al. (2008) to determine the X-ray area curves. For the high-luminosity sample (Section 2.2.5), the equivalent selection function is simply a top-hat function over the entire redshift range $(1.9<z<3.5)$, corresponding to a complete spectroscopic followup of the purely X-ray-selected high-luminosity sample.

For each of the individual colour-selected sources, we must assume a redshift probability distribution, $p_{i}(z)$, for which we adopt the selection function for the appropriate field and sample (unless a spectroscopic redshift is available in which case we adopt a delta function). Thus the differing redshift coverage of the selection criteria for different types and filter sets will provide some additional information regarding the evolution, and this effect will be explicitly accounted for by our study. The X-ray flux uncertainty is also accounted for in an identical manner as for the low-redshift hardband-selected samples, only converting from the soft-band flux to 2-10 keV rest-frame luminosities, to determine $p\left(d_{i} \mid L_{X}, z\right)$ for each of our colour-selected objects.

Additional weighting factors, $w_{i}$, must also be introduced, which are added as exponents of the individual source likelihoods in equation (10). This factor serves two purposes. First, it reduces the weight assigned to sources in our hard X-ray samples according to the fraction of their $p(z)$ distribution which falls below $z<1.2$ :

$w_{i}=\frac{\int_{0}^{1.2} \mathrm{~d} z p_{i}(z)}{\int_{0}^{\infty} \mathrm{d} z p_{i}(z)}$.

Thus, sources will be assigned a weight of $w_{i}=1$ if they have a spectroscopic redshift $z_{\text {spec }}<1.2$ or if their $p_{i}(z)$ based on the photo- $z$ estimate falls wholly below $z=1.2$. Sources with $z_{\text {spec }}>$ 1.2 or $p_{i}(z)$ which is all above $z=1.2$ will be assigned a weight of 0 (effectively removing them). Sources with photo- $z$ estimates that span above and below $z=1.2$ will be given appropriate fractional weights.

For the colour pre-selected objects, $w_{i}$ is the ratio of the total comoving volume sampled, based on our $z>1.2$ estimate of the area curve (equation 11), to the effective comoving volume sampled, based on the selection function at the object's $\mathcal{R}$ magnitude:

$w_{i}=\frac{V_{\text {tot }}}{V_{\text {eff }}}=\frac{\int_{1.2}^{\infty} A\left(L_{i}, z\right) \frac{\mathrm{d} V}{\mathrm{~d} z} \mathrm{~d} z}{\sum_{\text {fields }} \int A_{f}\left(L_{i}, z\right) P_{f}\left(\mathcal{R}_{i}, z\right) \frac{\mathrm{d} V}{\mathrm{~d} z} \mathrm{~d} z}$,

where $P_{f}\left(\mathcal{R}_{i}, z\right)$ is the selection function for both BX and LBG selection for a field for the observed $\mathcal{R}$ magnitude. Thus for the highredshift sample, the $w_{i}$ factors apply additional weight to sources with fainter optical magnitudes, which were thus less likely to be selected by the colour pre-selection technique.

Our colour-selected sources are also incorporated into the calculation of the $f_{\mathrm{X}} / f_{\text {opt }}$ distribution and the subsequent completeness correction. The $f_{\mathrm{X}} / f_{\text {opt }}$ derived considers hard-band X-ray fluxes. To achieve this an equivalent hard-band flux is calculated for each $L_{\mathrm{X}}, z$, whereas $L_{\mathrm{X}}$ for a source was originally derived from the observed soft-band flux. $R$-band magnitudes are derived from the $\mathcal{R}$ magnitudes by applying a small colour correction (based on our model templates described above).

Sources identified as low-redshift interlopers (Section 2.2.4) are excluded from the colour-selected samples, but may still be included in the main sample to constrain the $z<1.2$ XLF for the AEGIS and CDF-N fields where our surveys overlap. 34 sources which were assigned photometric redshifts in our original hard X-ray-selected samples are also present in our BX or LBG samples. Of these, 17 were assigned $z_{\text {phot }}<1.2$; the remainder were assigned to the higher 
redshifts expected for objects in our colour-selected samples. For all of these sources, we do not consider the photo- $z$ distribution, and consider them solely with the colour-selected samples, assigning the associated redshift distribution (based on the optical selection functions) and weighting factors.

\subsection{Bayesian model comparison and parameter estimation}

Having determined the likelihood function, our knowledge of the XLF can be fully described by the posterior probability function:

$p\left(\theta, \mu, \sigma \mid\left\{d_{i}\right\}, H\right)=\frac{\mathcal{L}\left(\left\{d_{i}\right\} \mid \theta, \mu, \sigma\right) \pi(\theta, \mu, \sigma \mid H)}{p\left(\left\{d_{i}\right\} \mid H\right)}$,

where we have introduced the symbol $H$ to represent a particular model hypothesis for the XLF and $\pi(\theta, \mu, \sigma \mid H)$ is the prior probability we assign to values for the parameters $\theta$, for model $H$. The denominator, $p\left(\left\{d_{i}\right\} \mid H\right)$, is known as the Bayesian evidence, $\mathcal{Z}$, and normalizes the posterior.

There are now two tasks at hand. First, we wish to determine the best estimates of the parameters, $\theta$, for our model XLF and assign them an error. Standard Monte Carlo Markov Chain approaches can be used to draw samples from the posterior, and thus provide estimates of the parameters. Such approaches ignore the normalizing factor, $p\left(\left\{d_{i}\right\} \mid H\right)$, as it is independent of the parameters. Our second task is to compare different evolutionary models. The Bayesian model comparison is achieved by calculating the ratio of the posterior probabilities for different models (e.g. Kass \& Raftery 1995):

$\frac{p\left(H_{1} \mid\left\{d_{i}\right\}\right)}{p\left(H_{2} \mid\left\{d_{i}\right\}\right)}=\frac{p\left(\left\{d_{i}\right\} \mid H_{1}\right)}{p\left(\left\{d_{i}\right\} \mid H_{2}\right)} \frac{\pi\left(H_{1}\right)}{\pi\left(H_{2}\right)}=\frac{\mathcal{Z}_{1}}{\mathcal{Z}_{2}} \frac{\pi\left(H_{1}\right)}{\pi\left(H_{2}\right)}$,

where the ratio of the prior probabilities of the two different models, $\pi\left(H_{1}\right) / \pi\left(H_{2}\right)$, can be taken as unity if we have no prior knowledge which indicates we should favour one of the models. The evidence can be calculated by integrating the likelihood over the prior parameter space; thus,

$\mathcal{Z}=\int \mathrm{d}^{D} \theta \mathcal{L}\left(\left\{d_{i}\right\} \mid \theta\right) \pi(\theta \mid H)$,

where $D$ represents the number of dimensions for the integration (i.e. the number of parameters, now incorporating the nuisance parameters $\mu$ and $\sigma$ into the parameter set $\theta$ ). To perform this integration we have implemented the 'nested sampling' algorithm (Skilling 2004), which provides an estimate of the evidence, $\mathcal{Z}$, along with a numerical uncertainty. 800 'live' objects are used for the nested sampling to ensure that the large, multidimensional parameter space is well sampled. Estimates of the posterior distribution may be recovered as a by-product of the nested sampling procedure, and thus used to determine the values of the parameters describing the XLF and their errors. We choose to report the posterior mode (equivalent to maximum likelihood) values of the parameters as our best estimates, and $1 \sigma$-equivalent (68.3 per cent) highest posterior density (HPD) intervals, which minimize the size of the confidence interval for the given confidence level.

It is worth noting the difference of this Bayesian model comparison approach to frequentist methods. The frequentist approach to comparing two models would be to calculate the likelihood ratio, the ratio of the maximum likelihood values for the two different models (Neyman \& Pearson 1928). The Bayesian approach, on the other hand, integrates the likelihood over the entire prior parameter space. As such, the Bayesian evidence incorporates knowledge of all the uncertainties in the data and the parameter estimation. This approach also has the advantage that it naturally implements Occam's Razor, favouring a simple model with a compact parameter space, thus less free parameters, over a more complicated one, unless the latter provides a significantly better description of the data (Berger \& Jefferys 1992).

\subsection{Binned estimates}

Our analysis of the evolution of the XLF considers each detected object individually and does not require any binning. Our results consist of our best-fitting parameter estimates and their uncertainties, and the relative Bayesian evidences for the different evolutionary models are presented in Section 4 and Tables 3 and 4. However, for display purposes it is useful to produce binned estimates which represent the value and uncertainties over certain discreet ranges of luminosities and redshifts. Our binned estimates are based on the $N_{\text {obs }} / N_{\text {mdl }}$ method (Miyaji, Hasinger \& Schmidt 2001), which multiplies the value of the best-fitting model evaluated at the centre of the bin by the ratio of the number of observed sources, $N_{\mathrm{obs}}$, to

Table 3. Best-fitting parameters and relative evidence for our different evolutionary models using the hard X-ray-selected sample only.

\begin{tabular}{lccccc}
\hline Parameter & Lower limit $^{a}$ & Upper limit & PLE & \multicolumn{1}{c}{ LADE } & \multicolumn{1}{c}{ LDDE } \\
\hline $\log K_{\text {norm }}\left(\right.$ or $\left.K_{0}\right) / \mathrm{Mpc}^{-3}$ & -7.0 & -3.0 & $-4.95_{-0.08}^{+0.08}$ & $-4.02_{-0.15}^{+0.15}$ & $-5.91_{-0.19}^{+0.19}$ \\
$\log L_{*}\left(\right.$ or $\left.L_{0}\right) / \mathrm{erg} \mathrm{s}^{-1}$ & 43.0 & 46.0 & $44.82_{-0.06}^{+0.06}$ & $44.59_{-0.12}^{+0.12}$ & $44.24_{-0.11}^{+0.11}$ \\
$\gamma_{1}$ & -0.1 & 1.5 & $0.70_{-0.03}^{+0.03}$ & $0.58_{-0.04}^{+0.04}$ & $0.80_{-0.03}^{+0.03}$ \\
$\gamma_{2}$ & 1.5 & 4.0 & $2.53_{-0.10}^{+0.10}$ & $2.55_{-0.12}^{+0.12}$ & $2.36_{-0.15}^{+0.15}$ \\
$p_{1}$ & 3.0 & 10.0 & $5.16_{-0.35}^{+0.35}$ & $6.60_{-0.58}^{+0.58}$ & - \\
$p_{2}$ & -4.0 & 3.0 & $-1.38_{-0.22}^{+0.22}$ & $0.63_{-0.42}^{+0.42}$ & - \\
$e_{1}$ & 2.0 & 6.0 & - & - & - \\
$e_{2}$ & -5.0 & 0.0 & - & - & $-2.88_{-0.30}^{+0.24}$ \\
$z_{c}\left(\right.$ or $\left.z_{c^{*}}\right)$ & 0.4 & 3.0 & $0.89_{-0.08}^{+0.08}$ & $0.75_{-0.14}^{+0.14}$ & $1.89_{-0.14}^{+0.14}$ \\
$d$ & -1.5 & 0.0 & - & $-0.36_{-0.03}^{+0.03}$ & - \\
$\log L_{a} / \mathrm{erg} \mathrm{s}^{-1}$ & 44.0 & 46.0 & - & - & $45.24_{-0.19}^{+0.19}$ \\
$\alpha$ & 0.0 & 1.0 & - & - & $0.15_{-0.01}^{+0.01}$ \\
$\Delta \ln \mathcal{Z}$ & & & $0.0 \pm 0.3$ & $+20.3 \pm 0.3$ & $+32.9 \pm 0.3$ \\
\hline
\end{tabular}

${ }^{a}$ Upper and lower limits on the constant priors for the parameters. 
Table 4. Best-fitting parameters and relative evidence for our evolutionary models using the hard-band-selected samples at $z<1.2$ and constrained at high redshifts using the colour pre-selected samples.

\begin{tabular}{lccc}
\hline Parameter & \multicolumn{1}{c}{ PLE } & \multicolumn{1}{c}{ LADE } & \multicolumn{1}{c}{ LDDE } \\
\hline $\log K_{\text {norm }}\left(\right.$ or $\left.K_{0}\right) / \mathrm{Mpc}^{-3}$ & $-5.10_{-0.04}^{+0.04}$ & $-4.53_{-0.07}^{+0.07}$ & $-6.08_{-0.06}^{+0.06}$ \\
$\log L *\left(\right.$ or $\left.L_{0}\right) / \mathrm{erg} \mathrm{s}^{-1}$ & $44.96_{-0.03}^{+0.03}$ & $44.77_{-0.06}^{+0.06}$ & $44.42_{-0.04}^{+0.04}$ \\
$\gamma_{1}$ & $0.70_{-0.02}^{+0.02}$ & $0.62_{-0.02}^{+0.02}$ & $0.77_{-0.01}^{+0.01}$ \\
$\gamma_{2}$ & $3.14_{-0.13}^{+0.13}$ & $3.01_{-0.11}^{+0.11}$ & $2.80_{-0.12}^{+0.12}$ \\
$p_{1}$ & $5.55_{-0.20}^{+0.20}$ & $6.36_{-0.40}^{+0.40}$ & - \\
$p_{2}$ & $-1.38_{-0.14}^{+0.14}$ & $-0.24_{-0.27}^{+0.27}$ & - \\
$e_{1}$ & - & - & $4.64_{-0.24}^{+0.24}$ \\
$e_{2}$ & - & - & $-1.69_{-0.12}^{+0.12}$ \\
$z_{c}\left(\right.$ or $\left.z_{c^{*}}\right)$ & $0.84_{-0.04}^{+0.04}$ & $0.75_{-0.09}^{+0.09}$ & $1.27_{-0.07}^{+0.07}$ \\
$d$ & - & $-0.19_{-0.02}^{+0.02}$ & - \\
$\log L_{a} / \mathrm{erg} \mathrm{s}^{-1}$ & - & - & $44.70_{-0.12}^{+0.12}$ \\
$\alpha$ & - & - & $0.11_{-0.01}^{+0.01}$ \\
$\Delta \ln \mathcal{Z}$ & $0.00 \pm 0.3$ & $+11.1 \pm 0.3$ & $+12.2 \pm 0.3$ \\
\hline
\end{tabular}

the predicted number in the bin, $N_{\text {mdl }}$, where

$N_{\mathrm{mdl}}=\int_{\Delta \log L_{\mathrm{X}}} \mathrm{d} \log L_{\mathrm{X}} \int_{\Delta z} \frac{\mathrm{d} V}{\mathrm{~d} z} A\left(L_{\mathrm{X}}, z\right) \phi\left(L_{\mathrm{X}}, z \mid \theta\right)$

for each bin of size $\Delta \log L_{\mathrm{X}}$ and $\Delta z$. With our methodology a source is assigned a distribution of luminosities and redshifts, and thus can be included in multiple bins. Therefore the observed number of sources, $N_{\mathrm{obs}}$, is found by summing the fractional contribution of all sources to each $\Delta L_{\mathrm{X}}-\Delta z$ bin:

$N_{\text {obs }}=\sum_{i=1}^{M} \frac{\int_{\Delta \log L_{\mathrm{X}}} \mathrm{d} \log L_{\mathrm{X}} \int_{\Delta z} \frac{\mathrm{d} V}{\mathrm{~d} z} p\left(d_{i} \mid L_{\mathrm{X}}, z\right)}{\int_{-\infty}^{\infty} \mathrm{d} \log L_{\mathrm{X}} \int_{0}^{\infty} \frac{\mathrm{d} V}{\mathrm{~d} z} p\left(d_{i} \mid L_{\mathrm{X}}, z\right)}$.

Errors on the binned values are based on approximate Poisson uncertainties on the effective number of objects in a bin. Points are only shown for bins with an effective $N_{\mathrm{obs}} \geq 1$.

\section{EVOLUTION OF THE XLF}

We have used our Bayesian methodology and the nested sampling algorithm to compare three different evolutionary models of the XLF, using both our hard X-ray-selected samples over all redshifts (using photometric and spectroscopic redshifts) and the combination of the hard X-ray sample at $z<1.2$ and the high-redshift colour pre-selected samples. For each evolutionary model we calculated the best fit (mode) for the parameters, with errors given by the HPD interval, and the relative logarithmic evidence, $\Delta \ln \mathcal{Z}$. Differences in $\ln \mathcal{Z} \gtrsim 4.6$ indicate very strong evidence in favour of the model with higher evidence (Jeffreys 1961). The results are given in Tables 3 and 4 for the hard X-ray sample only and the combined samples, respectively. Constant (or log-constant) priors were assumed for all parameters over the ranges indicated in Table 3.

\subsection{Evolutionary models}

We compare the following evolutionary models.

\subsubsection{Pure luminosity evolution}

At first we investigated models in which the XLF retains the same shape at all redshifts, but undergoes overall shifts in luminosity. We assume the standard double power-law shape for the XLF:

$\phi\left(L_{\mathrm{X}}\right)=\frac{\mathrm{d} \Phi\left(L_{\mathrm{X}}\right)}{\mathrm{d} \log L_{\mathrm{X}}}=K_{\mathrm{norm}}\left[\left(\frac{L_{\mathrm{X}}}{L_{*}}\right)^{\gamma_{1}}+\left(\frac{L_{\mathrm{X}}}{L_{*}}\right)^{\gamma_{2}}\right]^{-1}$,

where $\gamma_{1}$ is the faint-end slope, $\gamma_{2}$ is the bright-end slope, $L_{*}$ is the characteristic break luminosity and $K_{\text {norm }}$ is a normalization factor for the overall density. PLE can then be described as evolution of the characteristic luminosity, $L_{*}(z)$. Barger et al. (2005) found that the low-redshift $(z<1.2)$ XLF could be well described by a PLE model, where $L_{*}(z)$ is given by

$\log L_{*}(z)=\log L_{0}+p \log \left(\frac{1+z}{2}\right)$.

However, previous studies (e.g. Ueda et al. 2003; Aird et al. 2008; Silverman et al. 2008) have indicated that the strong evolution of $L_{*}$ at low redshifts may not continue above $z \sim 1$. A number of authors have utilized models which extend equation (20) with additional quadratic or higher order terms in $\log (1+z)$ (e.g. Page et al. 1997; Silverman et al. 2008). Alternatively, Ueda et al. (2003) described a PLE model which has two different slopes below and above a critical redshift, $z_{c}$. This parametrization allows the luminosity evolution to flatten off, cease completely, or increase or decline at high redshifts. We have adopted this form of luminosity evolution, additionally allowing for a smooth transition between the two slopes; thus, the evolution of $L_{*}$ is given by

$\log L_{*}(z)=\log L_{0}-\log \left[\left(\frac{1+z_{c}}{1+z}\right)^{p_{1}}+\left(\frac{1+z_{c}}{1+z}\right)^{p_{2}}\right]$,

where the $z_{c}$ parameter controls the transition from the strong low- $z$ evolution to the high- $z$ form.

\subsubsection{Luminosity and density evolution}

The work of Aird et al. (2008) indicated that while the XLF retained a similar shape at high redshift, there was an overall decline in the number density at the faint end ( $L_{\mathrm{X}} \lesssim L_{*}$ ), yet little or positive evolution at the bright end. This behaviour cannot be described by PLE. We have modified the PLE form described above to additionally allow for some overall decreasing density evolution with redshift, by allowing the normalization constant $K_{\text {norm }}$ to evolve as

$\log K_{\text {norm }}(z)=\log K_{0}+d(1+z)$,

thus introducing an additional parameter, $d$. With this parametrization, the shape of the XLF stays fixed at all redshifts; thus, our evolution consists of an XLF which shifts in both luminosity and overall density. We refer to this evolutionary model as LADE. This model is similar to the independent luminosity and density evolution (ILDE) described by Yencho et al. (2009), but with a different form for the density evolution and a more complex luminosity evolution that can flatten off at higher redshifts.

\subsubsection{Luminosity-dependent density evolution}

As discussed in Section 1, a number ofauthors have proposed that an evolutionary scheme in which the XLF undergoes a density evolution which is dependent on luminosity is required to accurately describe the evolution of both soft X-ray-selected (e.g. Miyaji, Hasinger \& Schmidt 2000; Hasinger, Miyaji \& Schmidt 2005) and hard X-ray-selected (e.g. Ueda et al. 2003; Silverman et al. 2008) AGN. We have adopted the parametrization given by Ueda et al. (2003), in which a typical double power-law form (e.g. equation 19) 
of the XLF at $z=0$ is modified by an evolution term which is a function of luminosity and redshift:

$\psi\left(L_{\mathrm{X}}, z\right)=\psi\left(L_{\mathrm{X}}, 0\right) e\left(L_{\mathrm{X}}, z\right)$,

where $e\left(L_{\mathrm{X}}, z\right)$ is a power-law function of $z$, which changes between two different forms at a cut-off redshift, $z_{c}$, which depends on the luminosity. Thus

$e\left(z, L_{\mathrm{X}}\right)=\left\{\begin{array}{ll}(1+z)^{e_{1}} & {\left[z<z_{c}\left(L_{\mathrm{X}}\right)\right]} \\ e\left(z_{c}\right)\left(\frac{1+z}{1+z_{c}\left(L_{\mathrm{X}}\right)}\right)^{e_{2}} & {\left[z \geq z_{c}\left(L_{\mathrm{X}}\right)\right]}\end{array}\right.$,

where

$z_{c}\left(L_{\mathrm{X}}\right)=\left\{\begin{array}{ll}z_{c *} & {\left[L_{\mathrm{X}} \geq L_{a}\right]} \\ z_{c *}\left(\frac{L_{\mathrm{X}}}{L_{a}}\right)^{\alpha} & {\left[L_{\mathrm{X}}<L_{a}\right]}\end{array}\right.$.

We allow all the parameters to vary in our fitting (although apply priors over constant ranges); thus the evidence, $\mathcal{Z}$, will fully account for the complexity of this parametrization.

\subsection{Results}

Table 3 gives the best-fitting (posterior mode) parameters for our three evolutionary models using the hard X-ray-selected samples only over all redshifts and values of the Bayesian evidence relative to the evidence for the PLE model, $\Delta \ln \mathcal{Z}$. These values indicate that the LADE model, introducing an additional parameter, provides a significantly better fit than the PLE model. There is also very strong evidence favouring the more complicated LDDE parametrization over either the LADE or PLE models. Fig. 8 plots the maximum-likelihood LDDE model along with binned estimates for a range of redshifts. It is immediately apparent that our LDDE model predicts a much milder evolution of the faint end of the XLF than the model presented by Silverman et al. (2008), with significantly less flattening of the faint-end slope. This is most likely due to our inclusion of objects with fainter optical magnitudes and correction for the remaining incompleteness in the samples (see further discussion in Section 5.1). However, the majority of the flattening of the faint-end slope we do observe is found at $z \gtrsim 1.2$, precisely where our photometric redshifts become unreliable and prone to catastrophic failure, and thus this measurement of the faint end of the XLF will be biased. Therefore, we are unable to confidently rule out the PLE or LADE evolutionary models using the results from direct hard X-ray selection only.

In Table 4 we present the results for the three evolutionary models using the colour pre-selected samples at high redshifts in addition to the hard X-ray samples at $z<1.2$. The colour pre-selection approach restricts the sample to sources for which we can reliably estimate the redshift. This should therefore provide more robust measurements of the XLF at high redshifts that are not subject to systematic biases due to catastrophic failure of photometric redshifts. The sample size is smaller and incomplete, but due to the well-defined selection functions we are able to correct for this incompleteness. We find strong evidence favouring the LADE or LDDE models over the PLE model. We can thus rule out this simplest model, consisting

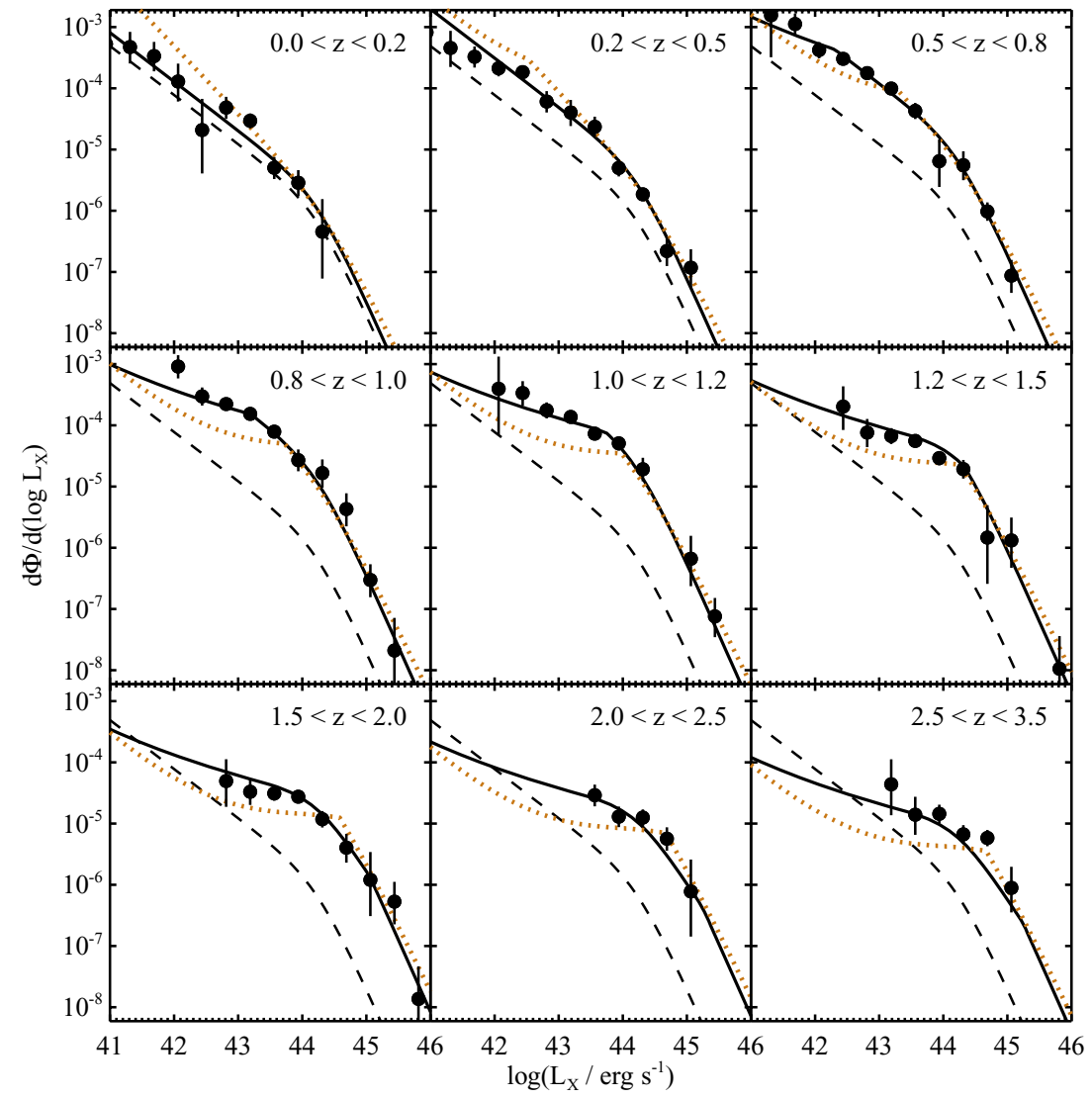

Figure 8. Our best-fitting LDDE model of the XLF using the hard X-ray-selected samples only, evaluated at the centres of the displayed redshift bins (solid lines), along with binned estimates (circles; see Section 3.5). The LDDE model evaluated at $z=0$ is shown in every panel (dashed lines). We compare our results to the LDDE model presented by Silverman et al. (2008) (dotted lines), which exhibits significantly greater flattening of the faint-end slope at high redshifts. However, we also note that our model fit will be biased at $z \sim 1.2-3$ where our photometric redshift estimates are prone to catastrophic failure. 
of an XLF which only evolves in luminosity without changing the shape or moving in overall density, and is thus unable to reproduce the lack of evolution of the bright end of the XLF at high redshifts and the observed decline at fainter luminosities. However, there is only very weak evidence ( $\Delta \ln \mathcal{Z}=+1.1$ ) favouring the most complex LDDE evolutionary scheme over the simpler LADE model in which the XLF retains the same shape at all redshifts. We therefore conclude that there is no significant evidence that an LDDE parametrization, and the associated flattening of the faint-end slope, is required to describe the evolution of the XLF. Our LADE model, in which the XLF retains the same shape but shifts in both luminosity and density, is also able to adequately describe the observed evolutionary behaviour. Our best-fitting LADE model indicates that strong luminosity evolution takes place between $z=0$ and $z=1$, but is consistent with a value of $p_{2}=0$, indicating that the evolution of $L_{*}$ flattens off at high redshifts, and the evolutionary behaviour becomes dominated by the overall negative density evolution.

Our LADE model is plotted in Fig. 9, evaluated at the centre of a number of redshift bins, along with binned estimates (see Section 3.5) using both the hard X-ray samples and the high- $z$ colour pre-selected samples. The LADE model is in good agreement with the bright end of the XLF determined from the hard X-ray-selected samples at $z>1.2$ (open circles, not used to constrain this model), but generally predicts a higher number density below $L_{*}$, reflecting the systematic failure of the photometric redshifts in this regime. At $2.0<z<2.5$, our binned estimates from the colour pre-selection are systematically below the LADE best fit (although agree within $\sim 1 \sigma$ ). Indeed, the binned data appear to indicate that the overall number density drops between $z \sim 1$ and $z \sim 2$, but then rises again between $z \sim 2$ and $z \sim 3$ (none of our evolutionary models could describe such behaviour). However, it is more likely that this behaviour reflects additional incompleteness in the BX selection, which has not been accounted for. The issues and limitations of our methodology are discussed further in Section 5.2. There is much better agreement with the $2.5<z<3.5$ binned estimates, where the sample is larger and the XLF is more robustly determined.

\section{DISCUSSION}

Our results for the evolution of the faint end of the $2-10 \mathrm{keV} \mathrm{XLF}$ should be the most accurate and robust to date. Our work utilizes both of the CDF, including the full $2 \mathrm{Ms}$ exposure available for each field. The additional large area of deep (200 ks) X-ray data provided by the AEGIS survey is also essential for an accurate determination of the faint-end slope. Our sensitive point source detection techniques allow us to probe the greatest depths in these data yet our Bayesian techniques allow us to fully account for uncertainty in the flux measurements at these faint limits, and account for the Eddington bias. By incorporating photometric redshift estimates we are able to achieve high-redshift completeness ( $\sim 75$ per cent), but we have accounted for the uncertainty in such redshift

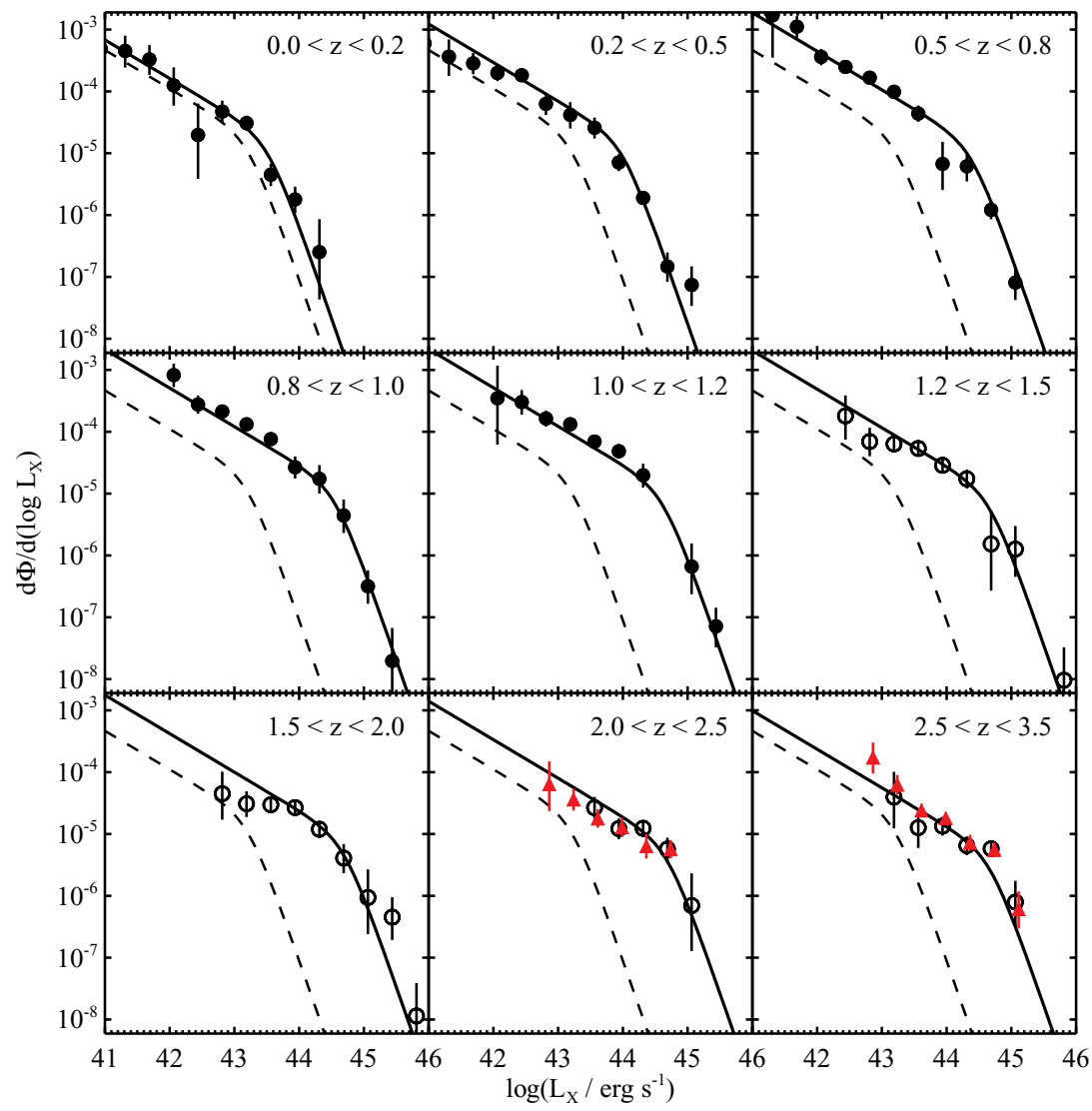

Figure 9. XLF results for the LADE model, constrained at high redshifts using the colour pre-selected samples as described in Section 3.3.2. Binned estimates at $z<1.2$ use the hard-band-selected sample only, including photometric redshift information (black circles). In the final two panels, binned estimates based on the high-redshift colour pre-selected samples that are used to constrain the evolution are shown (red triangles). At $z>1.2$, we also show binned estimates using the hard-band-selected sample, although these data were not used to fit the model (open circles). The LADE model evaluated at $z=0$ is shown in each panel (black dashed lines). 
determinations. We have corrected for the remaining incompleteness in our samples using the relation between optical and X-ray fluxes, which we have constrained based on our observed samples. At high redshifts, we use colour pre-selection to constrain the XLF and reduce biases due to the catastrophic failures of photo- $z$ estimates. We have also used a likelihood ratio matching technique to ensure that we only include robust optical counterparts to X-ray sources.

\subsection{Comparison with previous results}

Our work offers a number of improvements over previous studies. No previous work has considered the errors in X-ray fluxes and detection threshold, and thus will not account for the Eddington bias. Additionally, no prior studies utilizing photometric redshifts (e.g. Barger et al. 2005; Silverman et al. 2008; Brusa et al. 2009; Ebrero et al. 2009) have accounted for the known uncertainties in such estimates, or the possible effects of catastrophic failures which we believe led to a biased result at high redshifts in our work (see Fig. 8, Section 4.2). The commonly used photometric redshift catalogue for the CDF-N, presented by Barger et al. (2003), used the same optical data as our work, and the BPZ code, and thus these estimates will be subject to the same uncertainties and potential for catastrophic failures. Zheng et al. (2004) used a range of photo$z$ codes, incorporated a wider range of optical filters, deep HST imaging data and used the X-ray data as an indication of absorption properties, to provide improved photometric redshift estimates of Xray sources in the CDF-S (1 Ms data). Although these estimates were found to be accurate to within $\sim 8$ per cent, the authors discussed the remaining uncertainties, particularly for the fainter sources, and the possibility of catastrophic failure. The lack of spectroscopic followup makes it difficult to assess the reliability of all photometric redshifts at high- $z$, and the uncertainties should be accounted for in estimates of the XLF.

Our completeness corrections allow us to account for the lack of redshift information for a fraction of our sources, which is particularly important for determining the faint end of the XLF, where the optical counterparts are often too faint to be detected in even the deepest available imaging. Previous authors have often omitted any form of completeness correction, instead setting high X-ray flux limits (e.g. Ueda et al. 2003) or presenting maximal XLFs assigning all unidentified sources to a given redshift bin (e.g. Barger et al. 2005). Silverman et al. (2008) did apply a completeness correction, based on the fraction of sources with a given X-ray flux with redshift information, although samples were restricted to objects with bright optical counterparts $\left(r^{\prime}<24\right)$ which may introduce additional biases. Ebrero et al. (2009) restricted their analysis to samples with high-redshift identification completeness, and applied small additional corrections based on the fraction of identified sources as a function of the X-ray flux, although the completeness was likely to be overestimated in the CDF-S as they did not consider the high fraction of spurious counterparts. A similar approach was adopted by Yencho et al. (2009), although their samples were restricted to spectroscopic identifications and thus these completeness corrections were large ( $\sim 60$ per cent) at the faintest flux levels, and may be subject to additional biases and inaccuracies given the large number of complex factors involved in spectroscopic success rates. We thus believe our approach of considering only robust optical counterparts to X-ray sources and modelling the distribution of $f_{\mathrm{X}} / f_{\mathrm{opt}}$ ratios, in addition to our colour pre-selection approach at high redshifts, constitutes a more robust treatment of completeness than in previous work.

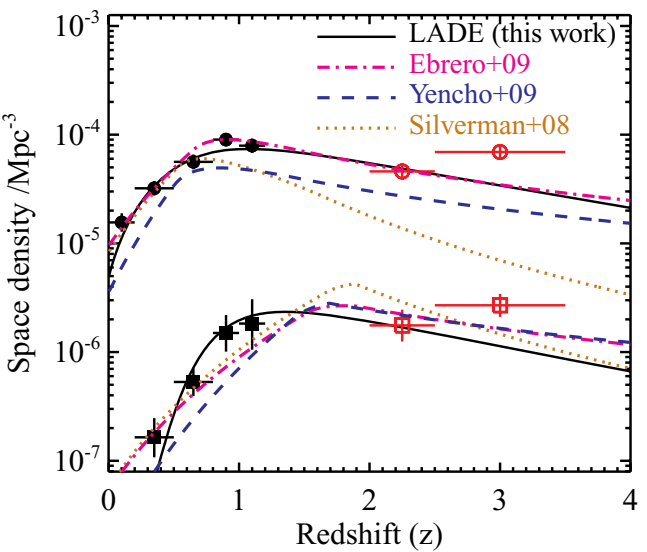

Figure 10. The evolution of the space density of AGN based on our LADE model for two luminosity ranges: $43<\log L_{X}<44.5$ (circles) and $44.5<\log L_{\mathrm{X}}<46$ (squares). Binned estimates are shown from the hard $\mathrm{X}$-ray samples at $z<1.2$ (solid symbols) and the colour pre-selected samples at $z \sim 2-3$ (open symbols). The space density based on LDDE models determined by Ebrero et al. (2009) (dot-dashed), Yencho et al. (2009) (dashed line) and Silverman et al. (2008) (dotted line) are also shown.

In Fig. 10 we directly compare the evolution of the space density of AGN predicted by our LADE model and a number of recent studies of the evolution of the XLF, all of which concluded that LDDE was taking place. Results are shown in two luminosity bins, which approximately correspond to above and below the characteristic break luminosity, $L_{*}$, although $L_{*}$ does evolve and thus such space density plots may provide a distorted view of the evolutionary behaviour of the XLF. Indeed, in the higher luminosity bin $(44.5<$ $\log L_{\mathrm{X}}<46$ ) our model indicates a very different evolution of the space density at $z \lesssim 1$ to previous (LDDE) results, but this is partly due to small offsets in the luminosity space which manifest as very large differences in density because of the steep slope of the bright end of the XLF. This can also lead to large differences in binned estimates depending on the methodology or assumed evolution of the XLF. However, in this regime, the XLF remains poorly constrained, and despite the very different evolutionary behaviour our binned results are consistent with the Silverman et al. (2008) and Ebrero et al. (2009) models at the $\sim 1 \sigma-2 \sigma$ level, although the Yencho et al. (2009) model is significantly lower. The high-redshift form of our evolution in this luminosity bin predicts space densities similar to the previous studies, although the form of the evolution and location of the peak in the number density are somewhat different.

Our results are in good agreement at low redshifts $(z \lesssim 1)$ in the lower luminosity bin $\left(43<\log L_{X}<44.5\right)$, although the Yencho et al. (2009) results remain below our estimates. However, at higher redshifts our model predicts a much higher space density than found by Yencho et al. (2009) or Silverman et al. (2008). We attribute the decline found by these authors to incompleteness, catastrophic failures of photometric redshift estimates and false associations of $\mathrm{X}$-ray sources with lower redshift optical counterparts. Conversely, the Ebrero et al. (2009) LDDE model is in very good agreement with our result, most likely because of the higher completeness of their samples. However, our results have shown that an LDDE model is not necessary to describe this evolution.

It is worth noting that our LADE form of the evolution is able to describe a 'cosmic-downsizing' behaviour in which the peak in the space density moves to lower redshifts for lower luminosity AGN, and indeed we do find small redshift offsets between the peak space 
densities for different luminosity ranges (e.g. Fig. 10). However, our model does not find the strongly luminosity-dependent shift in peak space density indicated by some prior studies (e.g. Hasinger et al. 2005; Silverman et al. 2008). This is not only due to our steeper faint-end slope (and thus higher number density of low-luminosity AGN at $z \gtrsim 1$ ), but also due to the differences in the form of the bright-end evolution discussed above.

\subsection{Remaining uncertainties}

While we believe our work offers a number of improvements over previous studies, there are remaining uncertainties associated with our approach.

\subsubsection{Photometric redshifts}

As previously discussed, the introduction of photometric redshifts, essential to improve the completeness of samples at faint X-ray fluxes, does introduce significant uncertainty, which we have tried to account for by considering probability distributions for the redshift estimates. However, there are a number of limitations to photometric redshift techniques that are thus also limitations in our studies. This is particularly apparent in the catastrophic failure of our photo$z$ estimates at $z \gtrsim 1.2$, which we believe led to a biased result for the evolution of the XLF, favouring an LDDE scheme with a flattening faint-end slope (Section 4.2, Table 3). Additionally, the majority of our photo- $z$ estimates are based on template fitting approaches that utilize a variety of galaxy templates (and priors based on the galaxy population), and thus the derived redshift probability distributions may not correspond to the true redshift for an AGN, which could bias our results at low as well as high redshifts. Incorporating AGN templates and using priors based on the AGN population may offer significant improvements and reduce systematic biases and catastrophic failures (e.g. Zheng et al. 2004; Salvato et al. 2009). Expanding the number and wavelength range of the filters, such as including near- and far-IR data, also has potential for improve photometric redshift estimates and provide better fits to AGN templates (e.g. Salvato et al. 2009).

In addition to the template fitting approach, we also utilized $\mathrm{ANN} z$ redshift estimates in the AEGIS field, in which an artificial neural network is trained to provide photometric redshifts using a sample of objects with spectroscopy. While this approach produced reliable results, it is limited to the subset of objects with a certain range of colours and magnitudes for which follow-up spectroscopy is available. Indeed, spectroscopic validation of photo- $z$ estimates for large numbers of optically faint X-ray sources is impossible with current instrumentation, which is a major limitation of photometric redshift techniques.

\subsubsection{High-z colour pre-selection}

To reduce the bias at high redshifts we incorporated rest-frame UV colour pre-selected samples. This should avoid the systematic biases associated with catastrophic failure of the photometric redshift estimates, but does risk introducing additional uncertainty and biases. As noted in Section 4.2, the binned estimates based on the colour pre-selected samples at $2.0<z<2.5$ (dominated by BX-selected objects) fell systematically below the model, ostensibly indicating a rise in the space density of AGN between $z \sim 2$ and $z \sim 3$ (also seen in Fig. 10). The BX criteria select objects in a very thin slice of colour space, identifying objects with a specific spectral shape rather than strong spectral features. This makes the $\mathrm{BX}$ selection both more susceptible to contamination by lower redshift sources and more prone to incompleteness than the more widely used LBG selection that identifies a strong break in the spectrum shortwards of the Lyman limit. The shape and efficiency of the derived selection functions will be highly dependent on the modelling of the AGN spectra and the scatter in their properties, and it is quite possible that our relatively simple modelling may have led to overestimates of the completeness. Indeed, both the LBG and BX selections require AGN to be bright in the rest-frame UV, and will therefore miss lowluminosity, moderately or heavily obscured AGN residing in red, evolved host galaxies. Aird et al. (2008) found that LBG selection recovered a number density of low-luminosity AGN that is at least as high as found via direct X-ray selection and follow-up, tentatively indicating that the AGN population at $z \sim 3$ is dominated by objects with blue colours (cf. $z \sim 1$; Nandra et al. 2007). However, this may not be true at slightly lower redshifts, resulting in a lower number density at $2.0<z<2.5$ via the $\mathrm{BX}$ selection as red galaxies will be missed.

As discussed in Section 2.2.4, our combination of LBG and X-ray selection to identify $z \sim 3$ AGN does not suffer from lowredshift contamination, but this is not the case for the BX selection. Without complete spectroscopic follow-up of the X-ray-detected BX candidates we cannot be confident that we have excluded all interlopers (although we believe we are able to identify low-redshift star-forming galaxies; see Fig. 4), and it is unclear how this might affect our derived evolution of the XLF. Extensions of the BX selection technique, which incorporate data at redder wavelengths, may allow low-redshift interlopers to be excluded, and help identify AGN in redder host galaxies. Indeed, a hybrid of the photometric redshift and colour pre-selection approaches, not only utilizing data from all available wavebands to perform SED fitting and estimate a redshift, but also performing a number of colour cuts to avoid catastrophic failures, and carefully modelling the distribution of AGN spectral properties to determine the incompleteness, may be the optimal approach to determining the high-redshift evolution.

It is also worth noting that our approach of combining direct hard $\mathrm{X}$-ray selection at $z<1.2$ and colour pre-selection at high redshifts provides no data for $z \approx 1.2-2.0$. Significant evolution may be taking place over this redshift range, particularly at high luminosities (e.g. Silverman et al. 2005). Indeed, at high luminosities incompleteness issues should be less severe, although we note that at $z \sim 2$ we expect that $\sim 10$ per cent of AGN with $L_{\mathrm{X}}=10^{44.5} \mathrm{erg} \mathrm{s}^{-1}$ will have optical magnitudes of $R>24$, based on our $f_{\mathrm{X}} / f_{\text {opt }}$ distributions. In this redshift range, photometric redshift estimates are prone to catastrophic failure, and spectroscopic identification can also be difficult even for bright targets. Thus, care must be taken to avoid biased results. As found in Section 4.2, such systematic biases can erroneously indicate the need for a more complex evolutionary model.

\subsubsection{Completeness corrections}

To account for objects with optical magnitudes below our limit of $R=25.5$, we introduced a simple completeness correction assuming that the $\log f_{\mathrm{X}} / f_{\text {opt }}$ distribution can be described by the same Gaussian function at all redshifts (Section 3.2). This may be an over simplification, as the redshift distribution of optically faint sources will differ from the brighter population. Indeed, Mainieri et al. (2005) found that optically faint X-ray sources tended to be found at higher redshifts. Additionally, Koekemoer et al. (2004) 
discussed how extreme X-ray objects (EXOs) which are X-ray bright but lack optical counterparts, even in very deep Hubble Advanced Camera for Surveys (ACS) imaging, may represent a population of very high-redshift $(z \sim 6-7)$ AGN where the redshifted Lyman break suppresses all emission at optical wavelengths. More sophisticated modelling of the rest-frame UV-optical properties of the AGN population and the effects on observed magnitudes as a function of redshift would enable improvements to our completeness corrections. Furthermore, the shape and evolution of the optical luminosity function of the AGN host galaxies will affect the completeness of our samples, could alter the shape and efficiency of the optical selection functions and may systematically impact the photo- $z$ estimates. In addition, we do not currently utilize any information from the optically unidentified population (their X-ray fluxes, the fact that they lack counterparts to the limits of our imaging), which could place further constraints on the evolution of the XLF.

\subsubsection{Cosmic variance}

Our investigation of the XLF uses an unprecedented large area of deep $200 \mathrm{ks}$ Chandra data in the AEGIS field, which supplements the data from the CDF used in previous studies (e.g. Barger et al. 2005; Silverman et al. 2008). As such, our determination of the faint-end evolution should be less susceptible to the effects of cosmic variance than in prior work. However, cosmic variance could introduce additional uncertainty in our XLF determinations (and thus weaken evidence for more complex evolutionary schemes). Indeed, assuming our X-ray-detected AGN cluster according to a power law with scalelength $r_{0}=7.1 \mathrm{Mpc}$ and slope $\gamma=1.8$ (typical of X-ray AGN at $z \sim 1$; Coil et al. 2009), we predict a fractional rms variation of $\sim 5-10$ per cent in the number density of AGN. This is comparable to our Poissonian uncertainties due to the number of objects in the bin although the errors will be basically covariant across the luminosity bins, and thus will not affect the shape of the XLF.

\subsubsection{Intrinsic absorption}

We have calculated rest-frame $2-10 \mathrm{keV}$ luminosities for our sources from their hard-band fluxes (or soft band for the high- $z$ sample), assuming a power law with $\Gamma=1.9$. This approach should correct for absorption effects due to intrinsic column densities of $N_{\mathrm{H}} \lesssim 10^{23} \mathrm{~cm}^{-2}$ at $z \sim 1$. However, the effectiveness of this correction varies with redshift, and this may bias our measurements of the luminosity and alter the effective sensitivity of our observations. The extent of these effects will depend on the distribution of AGN absorption properties, which may be evolving with redshift or have a luminosity dependence (Ueda et al. 2003; La Franca et al. 2005). Some previous studies (Ueda et al. 2003; La Franca et al. 2005; Ebrero et al. 2009) instead attempted to correct for the effects of absorption on a source-by-source basis and directly account for the $N_{\mathrm{H}}$ distribution in their calculation of the XLF. Determining the extent of absorption is difficult for our faint sources, which have few counts and thus little X-ray spectral information. The effects of absorption on our X-ray sensitivity and completeness calculations will be complicated, and should be corrected for a number of potential selection biases. Such effects are in fact likely to introduce considerable additional uncertainty as to the evolution of the faint end of the XLF, and could thus strengthen our assertions of the lack of a requirement for a more complicated LDDE model to describe the evolution of the XLF. We defer these considerations to future work.

\subsection{The evolution of AGN accretion activity}

Our determination of the evolution of the XLF presented in this paper sheds new light on how the distribution of AGN accretion activity evolves over the history of the Universe. We conclude that given the currently available data, the evolution of the XLF can be described by a LADE scheme, in which the XLF retains the same shape, but shifts in both luminosity and density with redshift. A more complicated luminosity-dependent scheme does not provide a significantly better description of the data; even so our best-fitting LDDE for the hard X-ray sample only, which we expect is biased at high redshifts, indicates a steeper faint-end slope and less evolution of the shape of the XLF than most previous work.

Hopkins et al. (2005) presented an interpretation of the AGN luminosity function in which the bright-end traces the mass distribution of black holes accreting at the peak rate over their lifetimes, while the faint end corresponds to AGN during transitionary periods as they approach or decline from their peak rate of activity. Furthermore, Hopkins et al. (2006) proposed that a luminosity-dependent lifetime for AGN could explain any observed flattening of the faintend slope, as less time is spent in the transitionary period by the more luminous AGN found at high redshifts. Our results, however, show that the shape of the XLF does not change; thus, a strongly luminosity-dependent lifetime may not be required. The lack of evolution in the shape of the XLF indicates that the processes involved in the triggering and feeding of AGN remain the same at all redshifts, but increase in overall density from the earliest times until $z \sim 1$, and move to lower luminosity systems between $z \approx 1$ and the present day. Such behaviour could be consistent with a mergerdriven model of the build-up of galaxy and black hole mass, and the triggering of AGN (e.g. Kauffmann \& Haehnelt 2000; Hopkins et al. 2008), in which the hierarchical build-up of galaxies increases the number density of AGN in the early history of the Universe, but the exhaustion of gas supplies in the most massive galaxies at later times (e.g. Cowie et al. 1996) results in the 'downsizing' of AGN to lower luminosities. Fully reconciling such a picture with the full range of observational data for AGN, in addition to the observed evolution of galaxies, using current theoretical models clearly requires significant further investigation. Indeed a number of other processes may play a role in triggering and fuelling low-luminosity AGN, such as minor mergers (Hernquist \& Mihos 1995), accretion of gas from a hot halo (Fabian \& Rees 1995) or bar-driven accretion (Sellwood \& Moore 1999). It is essential to assess the importance of such processes and understand the role they play in the evolution of AGN accretion activity.

In Fig. 11 we plot the $2-10 \mathrm{keV}$ luminosity density, $\int L_{\mathrm{X}} \phi\left(L_{\mathrm{X}}, z\right) \mathrm{d} \log L_{\mathrm{X}}$, as a function of redshift, which provides a tracer of the total AGN accretion activity. The evolution of the total luminosity density for all AGN with $L_{\mathrm{X}}>10^{42} \mathrm{erg} \mathrm{s}^{-1}$ is shown as well as the contribution of AGN in set luminosity ranges. The total luminosity density peaks at $z=1.2 \pm 0.1$, rapidly declining to lower redshifts, but the decline to higher redshifts is much milder indicating that significant AGN activity is taking place out to $z \sim$ $3-4$. AGN with luminosities in the range $L_{\mathrm{X}} \approx 10^{43-45} \mathrm{erg} \mathrm{s}^{-1}$ (i.e. $\sim L_{*}$ ) are responsible for the majority of the luminosity density, although the contribution of $L_{\mathrm{X}}=10^{44-45} \mathrm{erg} \mathrm{s}^{-1}$ AGN falls off rapidly at $z \lesssim 0.8$ as $L_{*}$ evolves, and $L_{\mathrm{X}}=10^{43-44} \mathrm{erg} \mathrm{s}^{-1}$ AGN dominate. 


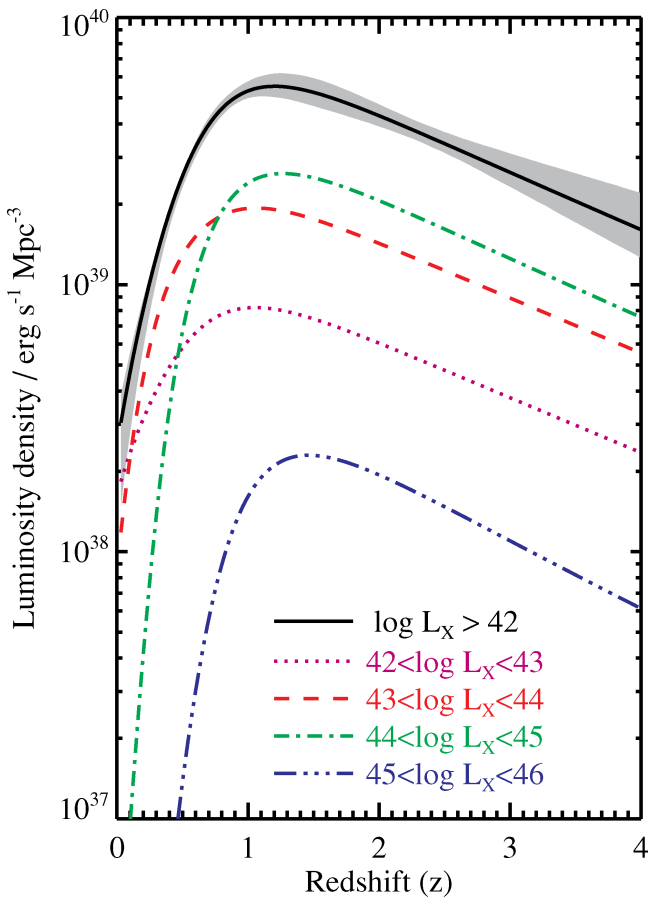

Figure 11. 2-10 keV luminosity density of AGN as a function of redshift for our LADE model integrated over the luminosity ranges indicated. The grey shaded region indicates the $1 \sigma$ uncertainty on the total luminosity density of AGN. The luminosity density is dominated by moderate luminosity $\left(43<\log L_{\mathrm{X}}<45\right)$ AGN and exhibits a mild decline above $z \approx 1.2$.

We can track the build-up of black hole mass more directly by relating AGN luminosity to mass accretion, as first proposed by Soltan (1982):

$L_{\mathrm{bol}}=\epsilon \dot{M_{\mathrm{acc}}} c^{2}=\frac{\epsilon \dot{M_{\mathrm{bh}}} c^{2}}{1-\epsilon}$,

where $\dot{M}_{\mathrm{acc}}$ is the mass accretion rate, $\dot{M}_{\mathrm{bh}}$ is the rate of change of black hole mass density, $L_{\mathrm{bol}}$ is the bolometric luminosity, $\epsilon$ is the radiative efficiency of the accretion process and $c$ is the speed of light. We have adopted a simple approach (e.g. Barger et al. 2005) by converting our 2-10 keV X-ray luminosities to bolometric values using a constant conversion factor (40; Elvis et al. 1994) and assuming a single value of the radiative efficiency, $\epsilon=0.1$ (Marconi et al. 2004). We note that a number of authors have discussed the need for luminosity-dependent bolometric corrections (e.g. Marconi et al. 2004; Hopkins, Richards \& Hernquist 2007), and Vasudevan \& Fabian (2007) reported significant object-to-object variation in bolometric corrections, which may depend on the Eddington ratio. The radiative efficiency may also vary significantly between AGN, depending on the spin of the black hole (e.g. Thorne 1974) in addition to the specifics of the accretion processes (e.g. Merloni $\&$ Heinz 2008). However, our simple assumptions allow an initial investigation of the consequences of our derived XLF evolution on the build-up of black hole mass. Using equation (26), we can convert our luminosity densities to a mass accretion rate and thus calculate the total black hole mass density as built up by accretion activity over the history of the Universe. Our results are shown in Fig. 12.

Based on our LADE model we predict a local black hole mass density of $2.2 \pm 0.2 \times 10^{5} \mathrm{M}_{\odot} \mathrm{Mpc}^{-3}$, where the error reflects the uncertainties in our model fit, but not the potentially larger uncertainties in bolometric correction or accretion efficiency. This

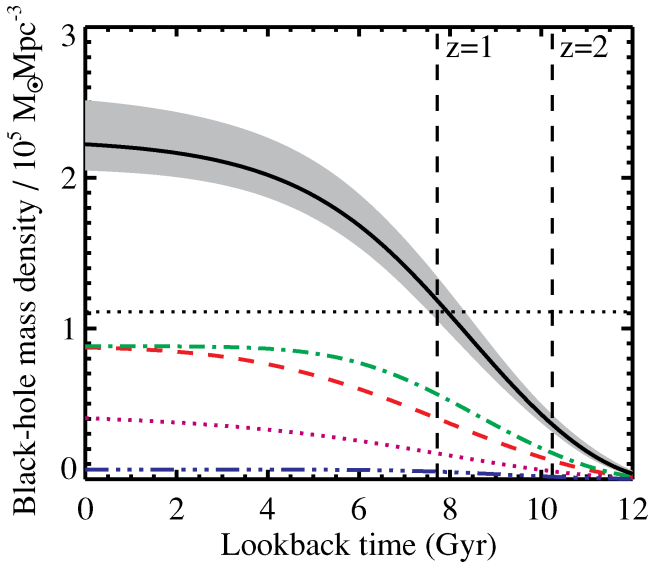

Figure 12. Total accreted black hole mass density against lookback time based on our LADE (the solid, grey shaded region indicates $1 \sigma$ uncertainty in the derived model). The lower curves correspond to the same luminosity ranges indicated in Fig. 11. We find that $\sim 50$ per cent of the local black hole mass density is built up in AGN actively accreting at $z \gtrsim 1$.

value is in good agreement with the estimate of $\mathrm{Yu} \&$ Tremaine (2002) $\left(2.5 \pm 0.4 \times 10^{5} \mathrm{M}_{\odot} \mathrm{Mpc}^{-3}\right)$ based on velocity dispersions of early-type galaxies in the Sloan Digital Sky Survey (SDSS) and the $M_{\mathrm{bh}}-\sigma$ relation, although it is lower than the estimate of Marconi et al. (2004) $\left(4.6_{-1.4}^{+1.9} \times 10^{5} \mathrm{M}_{\odot} \mathrm{Mpc}^{-3}\right)$, possibly indicating that our XLF does not provide a complete census of the history of accretion activity. Fig. 12 shows that a significant fraction ( $\sim 50$ per cent) of this total mass density is accreted at $z \gtrsim 1$. While the majority of the mass build up takes place in moderate luminosity $\operatorname{AGN}\left(L_{\mathrm{X}}=10^{43-45} \mathrm{erg} \mathrm{s}^{-1}\right)$, a significant fraction is accumulated at lower luminosities $\left(L_{X}=10^{42-43} \mathrm{erg} \mathrm{s}^{-1}\right)$. The LDDE model from Section 4 predicts a lower local black hole mass density, mainly due to the smaller numbers of AGN at these low luminosities and high redshifts. The redshift range of $z \sim 1-3$ clearly corresponds to a period of significant AGN activity, and thus it is essential to accurately measure the XLF down to $L_{\mathrm{X}} \approx 10^{42-43} \mathrm{erg} \mathrm{s}^{-1}$ in this epoch to determine the history of black hole mass accretion.

We can also compare our derived black hole mass accretion rates to star formation rates, which we show in Fig. 13. Previous studies (e.g. Boyle \& Terlevich 1998; Silverman et al. 2008) have shown close similarities between the rapid increase in the star formation rate and black hole accretion at $z \lesssim 1$, which we confirm. Our results indicate that this correlation continues out to high redshifts (cf. Silverman et al. 2008), at least when comparing to the recent star formation rates of Bouwens et al. (2007), although we note that our model is extrapolated far beyond the redshift range probed by our data. Comparisons of the galaxy and AGN luminosity functions may reveal differences in the details of the evolving distributions of activity, which could reveal further facets of the co-evolutionary processes and the feedback regulating AGN activity and star formation.

\section{SUMMARY}

We have presented new observational determinations of the XLF of AGN. We utilized a hard X-ray-selected sample from both the $2 \mathrm{Ms}$ CDF and the large area, deep $200 \mathrm{ks}$ Chandra survey in the AEGIS field. A likelihood ratio method was employed to match $\mathrm{X}$-ray sources to optical counterparts and ensure that only robust associations were considered. To improve our redshift completeness, we supplemented spectroscopic identifications with photometric 


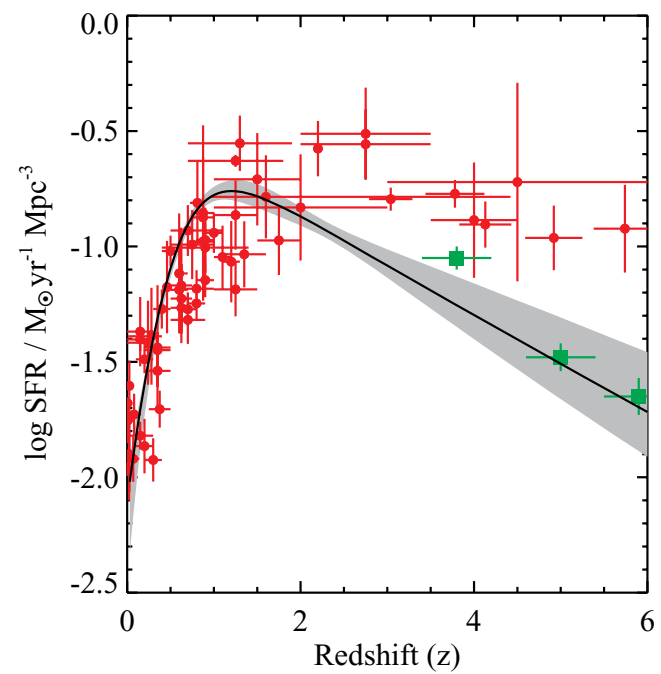

Figure 13. Comparison of star formation rates (circles) from the compilation of Hopkins (2004) and black hole accretion rate based on our LADE model (solid line; grey shading indicates $1 \sigma$ uncertainty in the model). The black hole mass accretion rate assumes a constant bolometric correction and accretion efficiency, $\epsilon=0.1$, and is scaled up by a factor of 5000 (Silverman et al. 2008). We also show recent measurements of the star formation rate at high redshifts from Bouwens et al. (2007) (squares), which indicate that the correlation between star formation and black hole accretion rate continues at high redshifts.

redshifts. However, we found our that photo- $z$ estimates were systematically biased and prone to catastrophic failure at $z \gtrsim 1.2$. We therefore adopted a rest-frame UV colour pre-selection approach to constrain the XLF at high redshifts. By carefully modelling the expected colour distributions of AGN, performing simulations of the optical data and repeating the source recovery procedure, we were able to determine well-defined selection functions that allowed us to correct for the incompleteness associated with the pre-selection technique.

We developed a sophisticated Bayesian methodology to determine the evolution of the XLF, which accounts for the uncertainties in photometric redshift estimates, the Poissonian nature of the $\mathrm{X}$-ray flux estimate, the fraction of sources with counterparts below the magnitude limits of the optical data and the optical selection functions at high redshifts. Using a Bayesian model comparison approach, we found that a PLE was unable to adequately describe the evolution of the XLF, but a scheme in which the XLF evolves in both luminosity and density, but does not change shape, did provide a good fit. We did not find significant evidence for a more complex LDDE and the associated flattening of the faint-end slope. However, an LDDE model was required if the direct X-ray-selected samples and the photometric redshift estimates were utilized at $z>$ 1.2 , but we believe such a result to be biased due to the inclusion of unreliable photo- $z$ estimates.

The form of our derived evolution of the XLF differs from many previous results (e.g. Silverman et al. 2008; Ebrero et al. 2009; Yencho et al. 2009). Our XLF retains the same shape at all redshifts, which may have a number of consequences for the evolution of the black hole mass function, AGN lifetimes and duty cycles, and the processes that fuel and trigger AGN. We find that the total luminosity density peaks at $z=1.2 \pm 0.1$. While there is a mild decline at $z>1.2$, significant AGN activity is still taking place at high redshifts. We find significantly higher number densities of low-luminosity $\mathrm{AGN}\left(L_{\mathrm{X}} \lesssim 10^{44} \mathrm{erg} \mathrm{s}^{-1}\right)$ at $z \gtrsim 1$, which make a significant contribution to the total luminosity density and growth of black hole mass at these redshifts. Our results do not indicate a strong shift of the peak space density to lower redshifts for lower luminosities, although a small shift is predicted by our bestfitting evolutionary model. The mild decline in AGN activity to high redshifts appears to correlate with that seen in the star formation rate, consistent with a co-evolutionary scheme for black holes and galaxies.

\section{ACKNOWLEDGMENTS}

We acknowledge financial support from STFC (JA, ESL). Some observations reported here were obtained at the MMT Observatory, a joint facility of the Smithsonian Institution and the University of Arizona. Also based in part on observations obtained with MegaPrime/MegaCam, a joint project of CFHT and CEA/DAPNIA, at the CFHT which is operated by the National Research Council (NRC) of Canada, the Institut National des Sciences de l'Univers of the Centre National de la Recherche Scientifique (CNRS) of France and the University of Hawaii. This work is based in part on data products produced at TERAPIX and the Canadian Astronomy Data Centre as part of the CFHTLS, a collaborative project of NRC and CNRS.

\section{REFERENCES}

Adelberger K. L., Steidel C. C., 2000, ApJ, 544, 218

Adelberger K. L., Steidel C. C., Shapley A. E., Hunt M. P., Erb D. K., Reddy N. A., Pettini M., 2004, ApJ, 607, 226

Aird J., Nandra K., Georgakakis A., Laird E. S., Steidel C. C., Sharon C., 2008, MNRAS, 387, 883

Akiyama M. et al., 2000, ApJ, 532, 700

Akiyama M., Ueda Y., Ohta K., Takahashi T., Yamada T., 2003, ApJS, 148, 275

Alexander D. M. et al., 2003, AJ, 126, 539

Barger A. J. et al., 2003, AJ, 126, 632

Barger A. J., Cowie L. L., Mushotzky R. F., Yang Y., Wang W.-H., Steffen A. T., Capak P., 2005, AJ, 129, 578

Barger A. J., Cowie L. L., Wang W.-H., 2008, ApJ, 689, 687

Barmby P., Huang J.-S., Ashby M. L. N., Eisenhardt P. R. M., Fazio G. G., Willner S. P., Wright E. L., 2008, ApJS, 177, 431

Benítez N., 2000, ApJ, 536, 571

Berger J., Jefferys W., 1992, Statistical Methods Applications, 1, 17

Bershady M. A., Charlton J. C., Geoffroy J. M., 1999, ApJ, 518, 103

Bouwens R. J., Illingworth G. D., Franx M., Ford H., 2007, ApJ, 670, 928

Boyle B. J., Terlevich R. J., 1998, MNRAS, 293, L49

Boyle B. J., Griffiths R. E., Shanks T., Stewart G. C., Georgantopoulos I., 1993, MNRAS, 260, 49

Brusa M. et al., 2009, ApJ, 693, 8

Bruzual G., Charlot S., 2003, MNRAS, 344, 1000

Calzetti D., Armus L., Bohlin R. C., Kinney A. L., Koornneef J., StorchiBergmann T., 2000, ApJ, 533, 682

Capak P. et al., 2004, AJ, 127, 180

Ciliegi P., Zamorani G., Hasinger G., Lehmann I., Szokoly G., Wilson G., 2003, A\&A, 398, 901

Coil A. L. et al., 2009, ApJ, 701, 1484

Coleman G. D., Wu C.-C., Weedman D. W., 1980, ApJS, 43, 393

Collister A. A., Lahav O., 2004, PASP, 116, 345

Cowie L. L., Songaila A., Hu E. M., Cohen J. G., 1996, AJ, 112, 839

Cowie L. L., Barger A. J., Bautz M. W., Brandt W. N., Garmire G. P., 2003, ApJL, 584, L57

Davis M. et al., 2007, ApJ, 660, L1

Dickey J. M., Lockman F. J., 1990, ARA\&A, 28, 215

Dickinson M., Giavalisco M., The Goods Team, 2003, in Bender R., Renzini A., eds, The Mass of Galaxies at Low and High Redshift. SpringerVerlag, Berlin, p. 324 
Ebrero J. et al., 2009, A\&A, 493, 55

Elvis M. et al., 1994, ApJS, 95, 1

Fabian A. C., Rees M. J., 1995, MNRAS, 277, L55

Georgakakis A., Nandra K., Laird E. S., Aird J., Trichas M., 2008, MNRAS, 388,1205

Giacconi R. et al., 2002, ApJS, 139, 369

Hasinger G., Miyaji T., Schmidt M., 2005, A\&A, 441, 417

Hernquist L., Mihos J. C., 1995, ApJ, 448, 41

Hopkins A. M., 2004, ApJ, 615, 209

Hopkins P. F., Hernquist L., Cox T. J., Di Matteo T., Robertson B., Springel V., 2005, ApJ, 630, 716

Hopkins P. F., Hernquist L., Cox T. J., Robertson B., Di Matteo T., Springel V., 2006, ApJ, 639, 700

Hopkins P. F., Richards G. T., Hernquist L., 2007, ApJ, 654, 731

Hopkins P. F., Hernquist L., Cox T. J., Kereš D., 2008, ApJS, 175, 356

Hunt M. P., Steidel C. C., Adelberger K. L., Shapley A. E., 2004, ApJ, 605, 625

Ilbert O. et al., 2006, A\&A, 457, 841

Jeffreys H., 1961, Theory of Probability. Clarendon Press, Oxford

Kass R. E., Raftery A. E., 1995, J. American Statistical Association, 90, 773

Kauffmann G., Haehnelt M., 2000, MNRAS, 311, 576

Kinney A. L., Calzetti D., Bohlin R. C., McQuade K., Storchi-Bergmann T., Schmitt H. R., 1996, ApJ, 467, 38

Koekemoer A. M. et al., 2004, ApJ, 600, L123

Kron R. G., 1980, ApJS, 43, 305

La Franca F. et al., 2005, ApJ, 635, 864

Laird E. S. et al., 2009, ApJS, 180, 102

Le Fèvre O. et al., 2004, A\&A, 428, 1043

Loredo T. J., 2004, in Fischer R., Preuss R., Toussaint U. V., eds, AIP Conf. Ser. Vol. 735, 24th International Workshop on Bayesian Inference and Maximum Entropy Methods in Science and Engineering. Am. Inst. Phys., New York, p. 195

Luo B. et al., 2008, ApJS, 179, 19

Mainieri V. et al., 2005, A\&A, 437, 805

Marconi A., Risaliti G., Gilli R., Hunt L. K., Maiolino R., Salvati M., 2004, MNRAS, 351, 169

Merloni A., Heinz S., 2008, MNRAS, 388, 1011

Mignoli M. et al., 2005, A\&A, 437, 883

Miyaji T., Hasinger G., Schmidt M., 2000, A\&A, 353, 25

Miyaji T., Hasinger G., Schmidt M., 2001, A\&A, 369, 49

Mobasher B. et al., 2004, ApJ, 600, L167

Nandra K. et al., 2005, MNRAS, 356, 568

Nandra K. et al., 2007, ApJ, 660, L11

Neyman J., Pearson E. S., 1928, Biometrika, 20A, 175
Page M. J., Mason K. O., McHardy I. M., Jones L. R., Carrera F. J., 1997, MNRAS, 291, 324

Popesso P. et al., 2009, A\&A, 494, 443

Ravikumar C. D. et al., 2007, A\&A, 465, 1099

Reddy N. A., Steidel C. C., Erb D. K., Shapley A. E., Pettini M., 2006, ApJ, 653,1004

Reddy N. A., Steidel C. C., Pettini M., Adelberger K. L., Shapley A. E., Erb D. K., Dickinson M., 2008, ApJS, 175, 48

Salvato M. et al., 2009, ApJ, 690, 1250

Sargent W. L. W., Steidel C. C., Boksenberg A., 1989, ApJS, 69, 703

Sellwood J. A., Moore E. M., 1999, ApJ, 510, 125

Silverman J. D. et al., 2005, ApJ, 624, 630

Silverman J. D. et al., 2008, ApJ, 679, 118

Skilling J., 2004, in Fischer R., Preuss R., Toussaint U. V., eds, AIP Conf. Proc. Vol. 735, 24th International Workshop on Bayesian Inference and Maximum Entropy Methods in Science and Engineering. Am. Inst. Phys., New York, p. 395

Soltan A., 1982, MNRAS, 200, 115

Steidel C. C., Hamilton D., 1993, AJ, 105, 2017

Steidel C. C., Adelberger K. L., Giavalisco M., Dickinson M., Pettini M., 1999, ApJ, 519, 1

Steidel C. C., Hunt M. P., Shapley A. E., Adelberger K. L., Pettini M., Dickinson M., Giavalisco M., 2002, ApJ, 576, 653

Steidel C. C., Adelberger K. L., Shapley A. E., Pettini M., Dickinson M., Giavalisco M., 2003, ApJ, 592, 728

Stern D. et al., 2002, AJ, 123, 2223

Sutherland W., Saunders W., 1992, MNRAS, 259, 413

Szokoly G. P. et al., 2004, ApJS, 155, 271

Thorne K. S., 1974, ApJ, 191, 507

Trouille L., Barger A. J., Cowie L. L., Yang Y., Mushotzky R. F., 2008, ApJS, 179, 1

Ueda Y. et al., 1999, ApJ, 518, 656

Ueda Y., Ishisaki Y., Takahashi T., Makishima K., Ohashi T., 2001, ApJS, 133,1

Ueda Y., Akiyama M., Ohta K., Miyaji T., 2003, ApJ, 598, 886

Vanzella E. et al., 2008, A\&A, 478, 83

Vasudevan R. V., Fabian A. C., 2007, MNRAS, 381, 1235

Weiner B. J. et al., 2006, ApJ, 653, 1027

Wolf C. et al., 2004, A\&A, 421, 913

Yencho B., Barger A. J., Trouille L., Winter L. M., 2009, ApJ, 698, 380

Yu Q., Tremaine S., 2002, MNRAS, 335, 965

Zheng W. et al., 2004, ApJS, 155, 73

This paper has been typeset from a $\mathrm{T}_{\mathrm{E}} \mathrm{X} / \mathrm{L} \mathrm{T} \mathrm{E} \mathrm{X}$ file prepared by the author. 